\title{
D-branes Wrapped on Fuzzy del Pezzo Surfaces
}

\author{
Kazuyuki Furuuchi ${ }^{1}$ and Kazumi Okuyama ${ }^{2}$ \\ ${ }^{1}$ National Center for Theoretical Sciences \\ National Tsing-Hua University, Hsinchu 30013, Taiwan, R.O.C. \\ furuuchi@phys.cts.nthu.edu.tw \\ ${ }^{2}$ Department of Physics, Shinshu University \\ Matsumoto 390-8621, Japan \\ kazumi@azusa.shinshu-u.ac.jp
}

\begin{abstract}
We construct classical solutions in quiver gauge theories on D0-branes probing toric del Pezzo singularities in Calabi-Yau manifolds. Our solutions represent D4-branes wrapped around fuzzy del Pezzo surfaces. We study the fluctuation spectrum around the fuzzy $\mathbb{C P}^{2}$ solution in detail. We also comment on possible applications of our fuzzy del Pezzo surfaces to the fuzzy version of F-theory, dubbed F(uzz) theory.
\end{abstract}

August 2010 


\section{Introduction}

It is well known that the worldvolume theory on D-branes becomes non-commutative when the non-zero $B$-field is threading the cycle on which the D-branes are wrapped [1]. Recently, it was argued that this type of non-commutative deformation of D-brane worldvolume theory may have interesting implication for the phenomenology of F-theory GUT models [2, 3: 4]. In 四, the F-theory GUT model based on the 7-branes wrapped on a fuzzy 4-cycle in a Calabi-Yau manifold was dubbed " $F(u z z)$ theory", and the fuzzy geometry was analyzed by quantizing the data of gauged linear sigma model. In the local approach of F-theory GUTs [5,6] (see [7] for a review) where the gravity can in principle be decoupled by taking the 4-dimensional Planck mass $M_{p l}$ to infinity, which amounts to considering a non-compact Calabi-Yau manifold, the possible configurations of 7-brane are quite restricted. It turns out that the 7-brane should wrap on a del Pezzo surface inside the Calabi-Yau manifold. Therefore, it will be interesting to analyze the D-branes wrapped around fuzzy del Pezzo surfaces.

In this paper, we construct fuzzy del Pezzo surfaces directly as classical solutions of quiver gauge theories which appear as worldline theories of D0-branes probing the del Pezzo surfaces. 1 . Our solutions represent a collection of D0-branes puffed up into D4-branes which wrap around a fuzzy del Pezzo surface. In our construction, some of the bi-fundamental fields, which correspond to arrows in the quiver diagram, represent the non-commutative coordinates of the del Pezzo surface. These non-commutative coordinates are realized as harmonic oscillators whose Fock space corresponds to a quantized version of the toric diagram of the del Pezzo surface. The $k$-th del Pezzo surface $d P_{k}$, the blow-up of $\mathbb{C P}^{2}$ at $k$ generic points, is toric only up to $k=3$, hence we will consider classical solutions describing D4-branes wrapped on fuzzy $d P_{k}$ with $k=0,1,2,3$. These solutions are constructed by taking the ranks of the gauge group at the nodes of the quiver different from each other, and this amounts to considering fractional branes [9, 10, 11, 12, 13] probing the del Pezzo singularity.

Our study is complimentary to the previous studies on the moduli space of quiver gauge theories realized on a single D-brane probing a toric singularity in a Calabi-Yau manifolds. In this case, the moduli space of the quiver gauge theory coincides with the

1 In our fuzzy del Pezzo surfaces, the non-commutativities are between holomorphic- and anti-holomorphic coordinates. Non-commutative Calabi-Yau manifolds with non-commutativities between holomorphic coordinates have been studied previously by some authors (see e.g. [8]). 
Calabi-Yau manifold itself [14,15, 16]. On the other hand, our D4-brane solutions wrap on a (fuzzy) four-cycle in the Calabi-Yau manifold, and typically they do not have moduli corresponding to moving in the directions in the Calabi-Yau manifold.

Branes wrapped on fuzzy $\mathbb{C P}^{2}$ have been studied in the context of IIB matrix model [17] or as a giant graviton [18]. Our construction of fuzzy $\mathbb{C P}^{2}$, or more generally fuzzy del Pezzo surfaces, is different from those studied in these references, in particular we start with a quiver gauge theory which does not contain Chern-Simons terms which could support the fuzzy 4-cycle via the Myers effect. Instead, our solutions use the bi-fundamental fields and can be regarded as a generalization of a similar construction of fuzzy spheres in [19,20,21]. Our construction also has a relation to the Beilinson's construction of stable vector bundles on $\mathbb{C P}^{2}$ [22]. The Beilinson quiver is obtained by deleting some of the arrows in the McKay quiver. In our solutions, some of the bi-fundamental fields are set to zero, which corresponds to deleting the arrows in the McKay quiver.

If we naively generalize our construction of fuzzy D4-branes and try to construct D7-branes wrapped on a del Pezzo surface by starting from the worldvolume theory of D3-branes instead of D0-branes, we encounter the problem of gauge anomaly in the 4dimensional theory on the D3-branes, since the ranks of the gauge group at different nodes are different. We will comment on how to circumvent this problem. After that, we will consider a possible application of our construction of D7-branes wrapped on a fuzzy del Pezzo surface to F(uzz) theory.

This paper is organized as follows. In section 2 we review the construction of D2branes wrapped on fuzzy $\mathbb{C P}^{1}$ as classical solutions of $A_{1}$ quiver gauge theories, as a warm-up for our study of D4-branes wrapped on fuzzy del Pezzo surfaces. In section 3 we construct classical solutions of quiver gauge theories on D0-branes probing $\mathbb{C}^{3} / \mathbb{Z}_{3}$ orbifold. Our solutions represent D4-branes wrapped on fuzzy $\mathbb{C P}^{2}$. In section 4 we study the KK spectrum on fuzzy $\mathbb{C P}^{2}$ and show that the higher KK modes are truncated by the effect of non-commutativity on fuzzy $\mathbb{C P}^{2}$. In section 5 , by generalizing the construction of fuzzy $\mathbb{C P}^{2}$ we construct classical solutions corresponding to D4-branes wrapped on fuzzy $d P_{k}$ for $k=1,2,3$. In section 6 we briefly mention possible applications of our fuzzy del Pezzo surfaces to F(uzz) theory, and we comment on the issue of gauge anomaly in the quiver gauge theories in 4 dimensions. Finally, we conclude with discussions in section 7. In addition, two appendices are included in this paper: Appendix $\mathrm{A}$ is a review of the computation of D-brane charges for the $\mathbb{C}^{3} / \mathbb{Z}_{3}$ quiver theory. In appendix $\mathrm{B}$ we discuss intersections of curves on fuzzy $\mathbb{C P}^{2}$.

2 Some of our solutions have some moduli left, as discussed in section 5 . 


\section{Review of D-Branes Wrapped on Fuzzy $\mathbb{C P}^{1}$}

In [19], fractional D-branes wrapped on $\mathbb{C P}^{1}$ in the $A_{1}$ ALE space were studied as classical solutions of the $A_{1}$ quiver gauge theory. It turned out that the resulting $\mathbb{C P}^{1}$ was non-commutative, reflecting the matrix nature of the underlying gauge theory. A similar configuration of D5-brane wrapping fuzzy $\mathbb{C P}^{1}$ also appears in the Klebanov-Witten theory [23,21], which describes the worldvolume theory of D3-branes on the conifold singularity [24]. In this section, we review the classical solution of $A_{1}$ quiver theory representing the D-branes wrapped on fuzzy $\mathbb{C P}^{1}$ as a warm up for the study of D4-branes wrapping a fuzzy 4-cycle.

The gauge theory on the D-branes probing the $\mathbb{C}^{2} / \mathbb{Z}_{2}$ orbifold singularity is summarized by the $A_{1}$ quiver diagram [14]. Namely, the gauge group is $U\left(n_{1}\right) \times U\left(n_{2}\right)$ associated to the two nodes of the $A_{1}$ quiver diagram and the arrows connecting the nodes represent the bi-fundamental chiral multiplets $A_{i}, B_{i}(i=1,2)$ which transform under the gauge group as $\left(n_{1}, \overline{n_{2}}\right)$ and $\left(\overline{n_{1}}, n_{2}\right)$, respectively. The quiver gauge theory for the pure D0branes on the $\mathbb{C}^{2} / \mathbb{Z}_{2}$ orbifold corresponds to the gauge group of equal rank for the two nodes, while the fractional branes wrapping the 2 -cycle $\mathbb{C P}^{1}$ is described by the same quiver gauge theory with unequal rank $n_{1} \neq n_{2}$.

Let us consider the (classical) vacuum of this quiver gauge theory. The D-term condition is

$$
\begin{aligned}
& \sum_{i=1,2}\left(A_{i} A_{i}^{\dagger}-B_{i}^{\dagger} B_{i}\right)=\zeta_{1} \mathbf{1}_{n_{1}} \\
& \sum_{i=1,2}\left(B_{i} B_{i}^{\dagger}-A_{i}^{\dagger} A_{i}\right)=\zeta_{2} \mathbf{1}_{n_{1}} .
\end{aligned}
$$

The F-term condition is solved by simply setting $B_{1}=B_{2}=0$. Then the above D-term condition becomes

$$
\sum_{i=1,2} A_{i} A_{i}^{\dagger}=\zeta_{1} \mathbf{1}_{n_{1}}, \quad-\sum_{i=1,2} A_{i}^{\dagger} A_{i}=\zeta_{2} \mathbf{1}_{n_{2}}
$$

To find a solution that represents a D2-brane wrapped on fuzzy $\mathbb{C P}^{1}$, it is convenient to introduce two independent oscillators $a_{1}, a_{2}$ obeying the usual commutation relations

$$
\left[a_{i}, a_{j}^{\dagger}\right]=\delta_{i j}, \quad\left[a_{i}, a_{j}\right]=\left[a_{i}^{\dagger}, a_{j}^{\dagger}\right]=0 \quad(i=1,2),
$$

and we take the Chan-Paton vector spaces $\mathbb{C}^{n_{1}}, \mathbb{C}^{n_{2}}$ as

$$
\mathbb{C}^{n_{1}}=\mathcal{F}_{N}^{(2)}, \quad \mathbb{C}^{n_{2}}=\mathcal{F}_{N+1}^{(2)} .
$$


Here $\mathcal{F}_{N}^{(2)}$ denotes the Fock space of two oscillators with the total occupation number fixed to $N$ :

$$
\mathcal{F}_{N}^{(2)}=\left\{\left|m_{1}, m_{2}\right\rangle=\frac{\left(a_{1}^{\dagger}\right)^{m_{1}}\left(a_{2}^{\dagger}\right)^{m_{2}}}{\sqrt{m_{1} ! m_{2} !}}|0\rangle, \quad m_{1}+m_{2}=N\right\} .
$$

For our choice of Chan-Paton spaces (2.4), the ranks of two gauge groups $n_{1}, n_{2}$ are given by

$$
n_{1}=\operatorname{dim} \mathcal{F}_{N}^{(2)}=N+1, \quad n_{2}=\operatorname{dim} \mathcal{F}_{N+1}^{(2)}=N+2 .
$$

Then the bi-fundamental matter $A_{i}(i=1,2)$ can be viewed as a linear map from $\mathcal{F}_{N+1}^{(2)}$ to $\mathcal{F}_{N}^{(2)}$ :

$$
\mathcal{F}_{N+1}^{(2)} \stackrel{A_{i}}{\longrightarrow} \mathcal{F}_{N}^{(2)}
$$

We can easily find a solution of (2.2) by identifying $A_{i}$ as the annihilation operator $a_{i}$ whose action is restricted to the space $\mathcal{F}_{N+1}^{(2)}$ :

$$
A_{i}=\left.c a_{i}\right|_{\mathcal{F}_{N+1}^{(2)}}
$$

where $c$ is a complex number. The relation between the constant $c$ and the FI-parameters $\zeta_{r}(r=1,2)$ is determined from (2.2) $3^{3}$. Using the relation 1

$$
\sum_{i=1}^{2} a_{i} a_{i}^{\dagger}=2+\sum_{i=1}^{2} a_{i}^{\dagger} a_{i}
$$

we find that the coefficient $c$ in the solution (2.8) and the FI-parameters are related by

$$
\zeta_{1}=|c|^{2}(N+2), \quad \zeta_{2}=-|c|^{2}(N+1) .
$$

One can check that the overall $U(1)$ of the gauge group $\prod_{r} U\left(n_{r}\right)$ is decoupled since

$$
\sum_{r=1}^{2} n_{r} \zeta_{r}=(N+1) \zeta_{1}+(N+2) \zeta_{2}=0
$$

3 When the gauge group is $S U\left(n_{1}\right) \times S U\left(n_{2}\right)$ as in the Klebanov-Witten theory, $|c|^{2}$ is not a parameter but the VEV of baryonic current $\operatorname{Tr}\left(A_{i} A_{i}^{\dagger}-B_{i}^{\dagger} B_{i}\right)$ 23, 21.

4 Throughout this paper we use the relation $\left[a_{i}, a_{j}^{\dagger}\right]=\delta_{i j}$ restricted to a finite dimensional space such as $\mathcal{F}_{N}^{(2)}$. This relation holds exactly on the finite dimensional space if we read it as $\left.\left.a_{i}\right|_{\mathcal{F}_{N+1}^{(2)}} a_{j}^{\dagger}\right|_{\mathcal{F}_{N}^{(2)}}-\left.\left.a_{j}^{\dagger}\right|_{\mathcal{F}_{N-1}^{(2)}} a_{i}\right|_{\mathcal{F}_{N}^{(2)}}=\delta_{i j} \mathbf{1}_{\mathcal{F}_{N}^{(2)}}$. In other words, this relation has the form $M_{i} N_{j}-$ $\widetilde{N}_{j} \widetilde{M}_{i}=\delta_{i j}$ where $M_{i}, \widetilde{M}_{i}, N_{j}, \widetilde{N}_{j}$ are finite dimensional matrices. Note that the usual argument for the impossibility of realizing the relation $\left[a_{i}, a_{j}^{\dagger}\right]=\delta_{i j}$ in a finite dimensional space by taking trace on both sides does not apply here since $M_{i} \neq \widetilde{M}_{i}, N_{j} \neq \widetilde{N}_{j}$. 
To see this solution describes fuzzy $\mathbb{C P}^{1}$, let us introduce generators of $s u(2)$ :

$$
J^{a}=\frac{1}{2} a_{i}^{\dagger} \sigma_{i j}^{a} a_{j},
$$

where $\sigma^{a}(a=1,2,3)$ denote the $2 \times 2$ Pauli matrices. We can easily show that the above $J^{a}$ satisfy the $s u(2)$ algebra $\left[J^{a}, J^{b}\right]=i \varepsilon^{a b c} J^{c}$, and the Casimir element is given in terms of the total number of oscillators $\widehat{N}=a_{1}^{\dagger} a_{1}+a_{2}^{\dagger} a_{2}$ :

$$
\sum_{a=1}^{3} J^{a} J^{a}=\frac{1}{4} \widehat{N}(\widehat{N}+2) .
$$

Fuzzy $\mathbb{C P}^{1}$ is defined by the matrix algebra acting on the Fock space $\mathcal{F}_{N}^{(2)}$, i.e.

$$
\mathbb{C P}_{N}^{1}=\operatorname{End}\left(\mathcal{F}_{N}^{(2)}\right)=\operatorname{Mat}(N+1, \mathbb{C})
$$

where $\operatorname{Mat}(d, \mathbb{C})$ denotes the space of $d \times d$ matrices. Note that $J^{a}$ in (2.12) restricted on $\mathcal{F}_{N}^{(2)}$ generates the algebra of $\mathbb{C P}_{N}^{1}$. More generally, the space of linear maps $\operatorname{Hom}\left(\mathcal{F}_{N}^{(2)}, \mathcal{F}_{N+m}^{(2)}\right)$ can be thought of as the quantization of the sections of the line bundle $\mathcal{O}_{\mathbb{P}^{1}}(m)$.

To summarize, the solution in (2.8) represents a D2-brane wrapped on fuzzy $\mathbb{C P}^{1}$, which can also be viewed as a collection of D0-branes puffed up into the D2-brane. As emphasized in [4], the Fock space $\mathcal{F}_{N}^{(2)}$ can be viewed as a set of "fuzzy points" on $\mathbb{C P}_{N}^{1}$.

\section{D-branes wrapped on fuzzy $\mathbb{C P}^{2}$}

In this section, generalizing the construction of fuzzy $\mathbb{C P}^{1}$ in the previous section, we will construct classical solutions of the quiver gauge theory on the D0-branes probing a $\mathbb{C}^{3} / \mathbb{Z}_{3}$ orbifold singularity, and show that our solutions represent D4-branes wrapped on fuzzy $\mathbb{C P}^{2}$.

\subsection{Quiver Gauge Theory for $\mathbb{C}^{3} / \mathbb{Z}_{3}$ Orbifold}

The worldline theory on the D0-branes probing $\mathbb{C}^{3} / \mathbb{Z}_{3}$ singularity is summarized by the quiver diagram of three nodes with the gauge group $U\left(n_{1}\right) \times U\left(n_{2}\right) \times U\left(n_{3}\right)$ (see Fig. 1a) [10,11]. Each pair of nodes are connected by three arrows, which correspond to the bifundamental chiral multiplets in 4-dimension dimensionally reduced to 1-dimension. We 
denote those chiral multiplets as $A_{i}, B_{i}, C_{i}(i=1,2,3)$, and they transform under the gauge group as

$$
\begin{aligned}
A_{i} & \in\left(n_{1}, \overline{n_{2}}, 1\right) \\
B_{i} & \in\left(1, n_{2}, \overline{n_{3}}\right) \\
C_{i} & \in\left(\overline{n_{1}}, 1, n_{3}\right) .
\end{aligned}
$$

The orbifold $\mathbb{C}^{3} / \mathbb{Z}_{3}$ can be thought of as a degenerate limit of the non-compact Calabi-Yau space $\mathcal{O}_{\mathbb{P}^{2}}(-3)$ where the base $\mathbb{C P}^{2}$ collapsed to a point. Therefore, we expect that the fractional D4-branes wrapped on vanishing $\mathbb{C P}^{2}$ can be described as a classical solution of this quiver gauge theory with an appropriate choice of $n_{1,2,3}$.

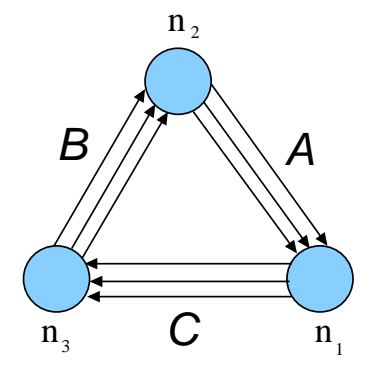

(a)

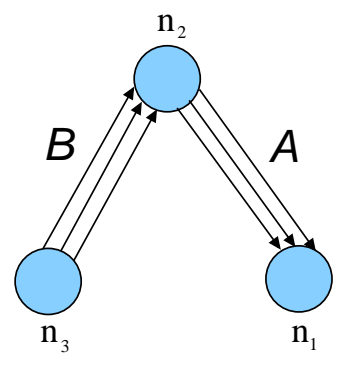

(b)

Fig. 1: (a) The McKay quiver for the worldline theory of D0-branes on $\mathbb{C}^{3} / \mathbb{Z}_{3}$ orbifold. (b) The Beilinson quiver describing vector bundles on $\mathbb{C P}^{2}$ is obtained by setting $C_{i}=0$ in the diagram (a).

Let us study the classical vacuum of this quiver gauge theory. The F-term condition coming from the superpotential $W=\varepsilon^{i j k} \operatorname{Tr}\left(A_{i} B_{j} C_{k}\right)$ reads

$$
A_{i} B_{j}=A_{j} B_{i}, \quad B_{i} C_{j}=B_{j} C_{i}, \quad C_{i} A_{j}=C_{j} A_{i} \quad(i \neq j)
$$

and the D-term condition is

$$
\begin{aligned}
& \sum_{i=1}^{3}\left(A_{i} A_{i}^{\dagger}-C_{i}^{\dagger} C_{i}\right)=\zeta_{1} \mathbf{1}_{n_{1}} \\
& \sum_{i=1}^{3}\left(B_{i} B_{i}^{\dagger}-A_{i}^{\dagger} A_{i}\right)=\zeta_{2} \mathbf{1}_{n_{2}} \\
& \sum_{i=1}^{3}\left(C_{i} C_{i}^{\dagger}-B_{i}^{\dagger} B_{i}\right)=\zeta_{3} \mathbf{1}_{n_{3}} .
\end{aligned}
$$

The last two equations in the F-term condition (3.2) are satisfied by setting $C_{i}=0$ for all $i=1,2,3$. This can be represented by deleting the arrows connecting the nodes 1 and 3 . 
The resulting quiver diagram with no arrows between the nodes 1 and 3 is known as the Beilinson quiver (see Fig. 1b), which was used to characterize the stable vector bundles on $\mathbb{C P}^{2}\left[22\right.$. After setting $C_{i}=0$, the F-term and D-term conditions for $A_{i}, B_{i}$ become

$$
\begin{gathered}
A_{i} B_{j}=A_{j} B_{i} \quad(i \neq j), \\
\sum_{i=1}^{3} A_{i} A_{i}^{\dagger}=\zeta_{1} \mathbf{1}_{n_{1}}, \quad \sum_{i=1}^{3}\left(B_{i} B_{i}^{\dagger}-A_{i}^{\dagger} A_{i}\right)=\zeta_{2} \mathbf{1}_{n_{2}}, \quad-\sum_{i=1}^{3} B_{i}^{\dagger} B_{i}=\zeta_{3} \mathbf{1}_{n_{3}} .
\end{gathered}
$$

In the next subsection, we will consider solutions of this set of equations.

\subsection{Solutions from the Beilinson Quiver}

We can consider various solutions of (3.4) by choosing $n_{1,2,3}$ appropriately. One interesting solution of (3.4) is found by taking

$$
\mathbb{C}^{n_{1}}=\mathcal{F}_{N}^{(3)}, \quad \mathbb{C}^{n_{2}}=\mathcal{F}_{N+1}^{(3)}, \quad \mathbb{C}^{n_{3}}=\mathcal{F}_{N+2}^{(3)},
$$

where $\mathcal{F}_{N}^{(3)}$ denotes the Fock space of three independent oscillators with total occupation number $N$ :

$$
\mathcal{F}_{N}^{(3)}=\left\{\left|m_{1}, m_{2}, m_{3}\right\rangle=\frac{\left(a_{1}^{\dagger}\right)^{m_{1}}\left(a_{2}^{\dagger}\right)^{m_{2}}\left(a_{3}^{\dagger}\right)^{m_{3}}}{\sqrt{m_{1} ! m_{2} ! m_{3} !}}|0\rangle, \quad m_{1}+m_{2}+m_{3}=N\right\} .
$$

The dimension of this space is

$$
\operatorname{dim} \mathcal{F}_{N}^{(3)}=\frac{1}{2}(N+1)(N+2)
$$

(3.7) can be counted from Fig. 2, which is a quantized version of the toric diagram of $\mathbb{C P}^{2}$. See Figure 3 of [25] for the toric diagram of commutative $\mathbb{C P}^{2}$.

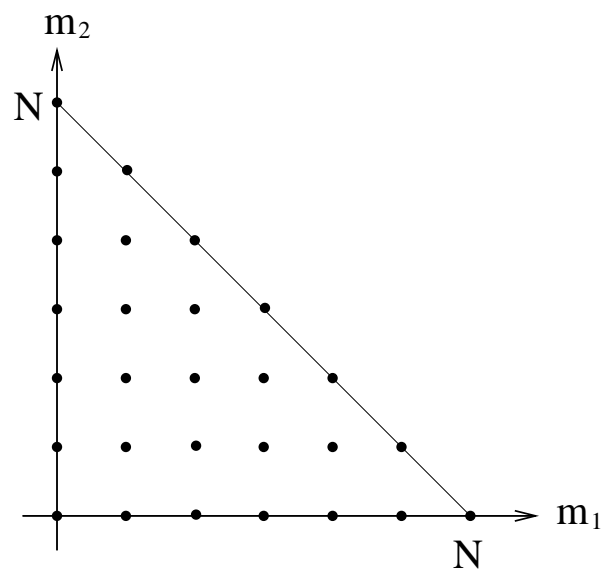

Fig. 2: "Quantized" toric diagram of fuzzy $\mathbb{C P}^{2} \cdot \mathbb{C P}^{2}$ is described as $S^{1} \times S^{1}$ fiber over the base space represented by the triangle of the toric diagram. The black points represent the points of the "quantized" base space of fuzzy $\mathbb{C P}^{2}$. The total number of the points in the quantized base space is finite. 
The dimension of $\mathcal{F}_{N}^{(3)}$ is given by the total number of the black points in Fig.2. As emphasized in $\left[4\right.$, fuzzy $\mathbb{C P}^{2}$ (or more precisely the base space when one sees $\mathbb{C P}^{2}$ as a torus fibered over the base space, as we will explain shortly) is made of finite number of points, which has many interesting consequences. Let us recall the description of commutative $\mathbb{C P}^{2}$ to see the correspondence with its fuzzy counterpart. The homogeneous coordinates $\left(z_{1}, z_{2}, z_{3}\right)$ on $\mathbb{C P}^{2}$ are subject to the equivalence relations

$$
\left(z_{1}, z_{2}, z_{3}\right) \sim\left(\lambda z_{1}, \lambda z_{2}, \lambda z_{3}\right)
$$

with $\lambda \in \mathbb{C}^{*}$. We can take the representative coordinates $\left(\Phi_{1}, \Phi_{2}, \Phi_{3}\right)$ from the equivalent class which satisfy

$$
\left|\Phi_{1}\right|^{2}+\left|\Phi_{2}\right|^{2}+\left|\Phi_{3}\right|^{2}=r^{2}
$$

for some fixed real number $r$. The generalization of (3.9) to fuzzy $\mathbb{C P}^{2}$ with the noncommutative coordinates $a_{i}$ corresponding to $\Phi_{i}(i=1,2,3)$ would be

$$
a_{1}^{\dagger} a_{1}+a_{2}^{\dagger} a_{2}+\left.a_{3}^{\dagger} a_{3}\right|_{\mathcal{F}_{N}^{(3)}}=m_{1}+m_{2}+m_{3}=N
$$

with fixed $N$, which appears in the definition (3.6). In the commutative case, we still have remaining equivalence relation through the phase:

$$
\left(\Phi_{1}, \Phi_{2}, \Phi_{3}\right) \sim\left(e^{i \theta} \Phi_{1}, e^{i \theta} \Phi_{2}, e^{i \theta} \Phi_{3}\right)
$$

with real number $\theta$. The non-commutative coordinates on fuzzy $\mathbb{C P}^{2}$ should be also identified through such equivalence relation. In our setting, this phase identification is provided by the $U(1)$ subgroups of the gauge groups at the nodes, as we will explain shortly.

From (3.7), $n_{1,2,3}$ in (3.5) are given by

$$
n_{1}=\frac{1}{2}(N+1)(N+2), \quad n_{2}=\frac{1}{2}(N+2)(N+3), \quad n_{3}=\frac{1}{2}(N+3)(N+4) .
$$

With this choice, $A_{i}$ 's and $B_{i}$ 's are interpreted as the linear maps between the Fock spaces:

$$
\mathcal{F}_{N+2}^{(3)} \stackrel{B_{i}}{\longrightarrow} \mathcal{F}_{N+1}^{(3)} \stackrel{A_{i}}{\longrightarrow} \mathcal{F}_{N}^{(3)}
$$

One obvious solution of (3.4) is obtained by setting $A_{i}$ and $B_{i}$ to be equal to the annihilation operator $a_{i}$ up to complex number coefficients $c, \widetilde{c}$ :

$$
A_{i}=\left.c a_{i}\right|_{\mathcal{F}_{N+1}^{(3)}}, \quad B_{i}=\left.\widetilde{c} a_{i}\right|_{\mathcal{F}_{N+2}^{(3)}}
$$


Then the F-term condition in (3.4) is automatically satisfied. The remaining D-term condition reads

$$
\begin{aligned}
|c|^{2} \sum_{i=1}^{3} a_{i} a_{i}^{\dagger} & =\zeta_{1} \mathbf{1}_{\mathcal{F}_{N}^{(3)}} \\
\sum_{i=1}^{3}\left(|\widetilde{c}|^{2} a_{i} a_{i}^{\dagger}-|c|^{2} a_{i}^{\dagger} a_{i}\right) & =\zeta_{2} \mathbf{1}_{\mathcal{F}_{N+1}^{(3)}} \\
-|\widetilde{c}|^{2} \sum_{i=1}^{3} a_{i}^{\dagger} a_{i} & =\zeta_{3} \mathbf{1}_{\mathcal{F}_{N+2}^{(3)}}
\end{aligned} .
$$

Using the relation

$$
\sum_{i=1}^{3} a_{i} a_{i}^{\dagger}=3+\sum_{i=1}^{3} a_{i}^{\dagger} a_{i}
$$

we find that $c, \widetilde{c}$ and the FI-parameters are related as

$$
\zeta_{1}=|c|^{2}(N+3), \quad \zeta_{2}=|\widetilde{c}|^{2}(N+4)-|c|^{2}(N+1), \quad \zeta_{3}=-|\widetilde{c}|^{2}(N+2)
$$

One can check that the above $\zeta_{r}$ satisfy

$$
\sum_{r=1}^{3} n_{r} \zeta_{r}=0
$$

which means that the overall $U(1)$ of the gauge group $\prod_{r} U\left(n_{r}\right)$ is decoupled. On the other hand, if we parametrize the $U(1)$ subgroup of $U\left(n_{r}\right)$ gauge group at the node $r$ by $e^{i \theta_{r}}(r=1,2,3)$, we obtain following gauge equivalence relations:

$$
A_{i} \sim e^{i\left(\theta_{3}-\theta_{2}\right)} A_{i}, \quad B_{i} \sim e^{\left(\theta_{2}-\theta_{1}\right)} B_{i} \quad(i=1,2,3)
$$

Since the overall $U(1)$ phase decouples, we have two independent $U(1)$ phases left, which we can choose as $\theta_{3}-\theta_{2}$ and $\theta_{2}-\theta_{1}$. (3.19) correspond to (3.11). More precisely, there are two coordinate system $A_{i}$ and $B_{i}$, and each of them has one equivalence relation by the $U(1)$ phase. Together with the restriction $m_{1}+m_{2}+m_{3}=N$ in (3.6), our construction of fuzzy $\mathbb{C P}^{2}$ can be regarded as a non-commutative version of the geometric visualization of $\mathbb{C P}^{2}$ from the toric diagram explained in [25]. To add some words for explanation, commutative $\mathbb{C P}^{2}$ is described as $S^{1} \times S^{1}$ fibration over the base represented by the edges and the inside of the triangle of the toric diagram, where $S^{1} \times S^{1}$ fiber is given by the 
relative phases of $\Phi_{i}$. The fiber degenerates at the edges of the triangle. In our fuzzy $\mathbb{C P}^{2}$, what is "quantized" is this base space, which is given by a finite set of points as above.5

The expected dimension of the solution space of (3.4) is given by

$$
d=3 n_{1} n_{2}+3 n_{2} n_{3}-\left(n_{1}^{2}+n_{2}^{2}+n_{3}^{2}-1\right)-3 n_{1} n_{3} .
$$

This can be understood as follows [11]. The first and the second terms represent the number of chiral multiplets $A_{i}, B_{i}$, and the middle term in the parenthesis is the dimension of the gauge group $\left[\prod_{r} U\left(n_{r}\right)\right] / U(1)$, and finally the last term in (3.20) is the number of F-term relations $A_{i} B_{j}=A_{j} B_{i}$. For our choice of $n_{1,2,3}$ in (3.5), we find $d=0$. Therefore, our solution corresponds to the "exceptional bundle" on $\mathbb{C P}^{2}$ [11].

Let us consider the D-brane charges carried by our solution. As reviewed in appendix A, the D-brane charge is related to the rank of gauge group $n_{1,2,3}$ by 6

$$
Q_{\mathrm{D} 4}=n_{1}-2 n_{2}+n_{3}, \quad Q_{\mathrm{D} 2}=n_{2}-n_{1}, \quad Q_{\mathrm{D} 0}=\frac{n_{1}+n_{2}}{2} .
$$

From (3.5), we find that the D-brane charges carried by our solution are given by

$$
Q_{\mathrm{D} 4}=1, \quad Q_{\mathrm{D} 2}=N+2, \quad Q_{\mathrm{D} 0}=\frac{1}{2}(N+2)^{2},
$$

and the BPS central charge is

$$
Z=Q_{\mathrm{D} 4}+Q_{\mathrm{D} 2} \omega+Q_{\mathrm{D} 0} \omega^{2}=e^{(N+2) \omega}
$$

where $\omega$ denotes the generator of $H^{2}\left(\mathbb{C P}^{2}\right)$. From (3.21) we see that our solution represents a single D4-brane wrapped on $\mathbb{C P}^{2}$ with $N+2$ unit of magnetic flux threading its worldvolume

$$
\frac{F}{2 \pi}=(N+2) \omega
$$

Due to this magnetic flux, the worldvolume theory on the D4-brane becomes noncommutative [1] with the non-commutativity parameter

$$
\theta=F^{-1} \sim \frac{1}{N+2}
$$

5 A construction of fuzzy toric geometries with a different procedure for quantizing the toric base has been investigated in [26].

6 In Fig. 1, we have reversed the direction of arrows compared to the diagram in [11], in order that $A_{i}, B_{i}$ correspond to the annihilation operator $a_{i}$, not the creation operator $a_{i}^{\dagger}$. Therefore, $X_{i}, Y_{i}$ in 11] correspond to our $A_{i}^{\dagger}, B_{i}^{\dagger}$, respectively. We have also changed the overall sign of the central charge from [11] so that our solution represents a D4-brane instead of a $\overline{\mathrm{D} 4}$-brane. 
We can also construct a solution describing $k$ D4-branes wrapping $\mathbb{C P}^{2}$ by tensoring the Chan-Paton spaces in $(3.5)$ with $\mathbb{C}^{k}$ :

$$
\mathbb{C}^{n_{1}}=\mathcal{F}_{N}^{(3)} \otimes \mathbb{C}^{k}, \quad \mathbb{C}^{n_{2}}=\mathcal{F}_{N+1}^{(3)} \otimes \mathbb{C}^{k}, \quad \mathbb{C}^{n_{3}}=\mathcal{F}_{N+2}^{(3)} \otimes \mathbb{C}^{k}
$$

and generalizing the single D4-brane solution (3.14) as

$$
A_{i}=c a_{i} \otimes \mathbf{1}_{k}, \quad B_{i}=\widetilde{c} a_{i} \otimes \mathbf{1}_{k} \quad(i=1,2,3)
$$

In particular, by taking $k=5$ we can construct a $U(5)$ gauge theory living on D7-branes, which may have useful applications in the model building in F(uzz) theory.

\subsection{Fuzzy $\mathbb{C P}^{2}$}

In this subsection, we will show that our solution (3.14) represents a BPS D4-brane wrapped on fuzzy $\mathbb{C P}^{2}$. One can repeat the analysis of fuzzy $\mathbb{C P}^{1}$ in the previous section by introducing the $s u(3)$ generators

$$
J^{a}=a_{i}^{\dagger} T_{i j}^{a} a_{j} \quad(a=1, \ldots, 8)
$$

where $T^{a}$ 's are the generators of $s u(3)$ in the fundamental representation. The commutation relations $\left[T^{a}, T^{b}\right]=i f^{a b c} T^{c}$ and $\left[a_{i}, a_{j}^{\dagger}\right]=\delta_{i j}$ imply $\left[J^{a}, J^{b}\right]=i f^{a b c} J^{c}$. By restricting the action of $J^{a}$ to the finite dimensional space $\mathcal{F}_{N}^{(3)}$, this construction leads to the fuzzy $\mathbb{C P}^{2}[27,28]$.

More generally, we can consider $n$ independent oscillators $\left[a_{i}, a_{j}^{\dagger}\right]=\delta_{i j}(i, j=1, \cdots, n)$, and construct the $s u(n)$ generators as $J^{a}=a_{i}^{\dagger} T_{i j}^{a} a_{j}$. Using the normalization $\operatorname{Tr}\left(T^{a} T^{b}\right)=$ $\frac{1}{2} \delta^{a b}$ and the identity

$$
T_{i j}^{a} T_{k l}^{a}=\frac{1}{2} \delta_{i l} \delta_{j k}-\frac{1}{2 n} \delta_{i j} \delta_{k l},
$$

one can show that $J^{a}=a_{i}^{\dagger} T_{i j}^{a} a_{j}$ satisfy

$$
\begin{aligned}
J^{a} J^{a} & =\frac{n-1}{2 n} \widehat{N}(\widehat{N}+n), \\
d_{a b c} J^{b} J^{c} & =\frac{n-2}{2 n}(2 \widehat{N}+n) J^{a} .
\end{aligned}
$$

Here $d_{a b c}=2 \operatorname{Tr}\left(T^{a}\left\{T^{b}, T^{c}\right\}\right)$ denotes the totally symmetric tensor and $\widehat{N}=\sum_{i} a_{i}^{\dagger} a_{i}$ is the total number of oscillators. 
We can define the fuzzy $\mathbb{C P}^{2}$ by

$$
\mathbb{C P}_{N}^{2}=\operatorname{End}\left(\mathcal{F}_{N}^{(3)}\right)=\operatorname{Mat}\left(\frac{1}{2}(N+1)(N+2), \mathbb{C}\right)
$$

and the generators of fuzzy $\mathbb{C P}_{N}^{2}$ algebra is $\left.J^{a}\right|_{\mathcal{F}_{N}^{(3)}}$. The relations satisfied by these generators are found by setting $n=3$ in (3.30):

$$
J^{a} J^{a}=\frac{1}{3} N(N+3), \quad d_{a b c} J^{b} J^{c}=\frac{1}{6}(2 N+3) J^{a} .
$$

Note that the value of $J^{a} J^{a}$ in 3.32 is the quadratic Casimir of the representation $\operatorname{Sym}^{N} \mathbf{3}=[N, 0]$ of $S U(3)$.

In the commutative language, our solution (3.14) corresponds to the line bundle $\mathcal{O}_{\mathbb{P}^{2}}(N+2)$, whose global sections are the degree $N+2$ polynomials. In the noncommutative setting, these sections correspond to the states in $\mathcal{F}_{N+2}^{(3)}$, which in turn is the largest space $\mathbb{C}^{n_{3}}$ in our quiver $(3.5)$.

\section{KK Spectrum on (Fuzzy) $\mathbb{C P}^{2}$}

In this section we study the KK spectrum on fuzzy $\mathbb{C P}^{2}$. We first review the spectrum of the scalar Laplacian on commutative $\mathbb{C P}^{2}$ and non-commutative $\mathbb{C P}^{2}$. Next we study the fluctuation spectrum of the gauge fields around our classical solution, and we will find that this spectrum agrees with the known eigenvalues of the scalar Laplacian on $\mathbb{C P}^{2}$ for the low-lying modes.

\subsection{Spectrum of the Scalar Laplacian on Commutative $\mathbb{C P}^{2}$}

Let us first recall the spectrum of the scalar Laplacian on commutative $\mathbb{C P}^{2}$ [29]. Since $\mathbb{C P}^{2}$ is a symmetric space of the form

$$
\mathbb{C P}^{2}=S U(3) / S(U(2) \times U(1))
$$

the Laplace equation $\Delta \phi=\lambda \phi$ for the scalar Laplacian

$$
\Delta=-\frac{1}{\sqrt{g}} \partial_{\mu}\left(g^{\mu \nu} \sqrt{g} \partial_{\nu}\right)
$$

can be solved by a group theoretical method. The Fubini-Study metric of $\mathbb{C P}^{2}$ is

$$
g_{i \bar{j}}=\partial_{i} \partial_{\bar{j}} K, \quad K=\frac{1}{M_{\mathrm{KK}}^{2}} \log \left(\left|z_{0}\right|^{2}+\left|z_{1}\right|^{2}+\left|z_{2}\right|^{2}\right),
$$


where $\left[z_{0}: z_{1}: z_{2}\right]$ are the homogeneous coordinates of $\mathbb{C P}^{2}$ and $1 / M_{\mathrm{KK}}$ sets the length scale of $\mathbb{C P}^{2}$. In terms of the inhomogeneous coordinates $w_{i}=\frac{z_{i}}{z_{0}}(i=1,2)$ on the patch $z_{0} \neq 0$, the Laplacian is written as

$$
\Delta=-4 M_{\mathrm{KK}}^{2}\left(1+\left|w_{1}\right|^{2}+\left|w_{2}\right|^{2}\right)\left[\sum_{i=1}^{2} \frac{\partial^{2}}{\partial w_{i} \partial \overline{w_{i}}}+\sum_{i, j=1}^{2} w_{i} \frac{\partial}{\partial w_{i}} \overline{w_{j}} \frac{\partial}{\partial \overline{w_{j}}}\right] .
$$

The eigenvalues of the scalar Laplacian are given by

$$
\lambda_{m}=4 M_{\mathrm{KK}}^{2} m(m+2), \quad(m=0,1,2, \cdots),
$$

and the multiplicity of the $m$-th eigenvalue is

$$
\mu_{m}=(m+1)^{3}
$$

This can be understood from the fact that the eigenspace associated with the $m$-th eigenvalue $\lambda_{m}$ transforms as the irreducible representation of $S U(3)$ with Dynkin label $[m, m]$. The dimension of the $[m, m]$ representation is $\mu_{m}$, and the eigenvalue $\lambda_{m}$ is proportional to the quadratic Casimir of the $[m, m]$ representation.

This eigenspace of the Laplacian is related to the space of homogeneous polynomials in the following way [30]. The space $\mathbb{C}\left[z_{i}\right]_{k}$ of degree $k$ polynomials in $z_{0}, z_{1}, z_{2}$ and its complex conjugate $\mathbb{C}\left[\overline{z_{i}}\right]_{k}$ transform under $S U(3)$ as $\operatorname{Sym}^{k} \mathbf{3}$ and $\operatorname{Sym}^{k} \overline{\mathbf{3}}$, respectively. Next we introduce the space of functions on $\mathbb{C P}^{2}$

$$
\mathcal{P}_{k}=\frac{\mathbb{C}\left[z_{0}, z_{1}, z_{2}\right]_{k} \otimes \mathbb{C}\left[\overline{z_{0}}, \overline{z_{1}}, \overline{z_{2}}\right]_{k}}{\left(\left|z_{0}\right|^{2}+\left|z_{1}\right|^{2}+\left|z_{2}\right|^{2}\right)^{k}}
$$

whose dimension is

$$
\operatorname{dim} \mathcal{P}_{k}=\left(\begin{array}{c}
k+2 \\
k
\end{array}\right)^{2}
$$

From the decomposition of $S U(3)$ representation

$$
\operatorname{Sym}^{k} \mathbf{3} \otimes \operatorname{Sym}^{k} \overline{\mathbf{3}}=\bigoplus_{m=0}^{k}[m, m]
$$

we find that the multiplicity of the $m$-th eigenvalue $\lambda_{m}$ is given by

$$
\mu_{m}=\operatorname{dim} \mathcal{P}_{m}-\operatorname{dim} \mathcal{P}_{m-1}=\left(\begin{array}{c}
m+2 \\
m
\end{array}\right)^{2}-\left(\begin{array}{c}
m+1 \\
m-1
\end{array}\right)^{2}=(m+1)^{3}
$$

Clearly, this agrees with (4.6). 


\subsection{Spectrum of the Scalar Laplacian on Fuzzy $\mathbb{C P}^{2}$}

As discussed in [31], the spectrum of the Laplacian on fuzzy $\mathbb{C P}_{N}^{2}$ is truncated by the total occupation number $N$ :

$$
\lambda_{m}=4 M_{\mathrm{KK}}^{2} m(m+2), \quad(m=0,1, \cdots, N) .
$$

This is because the space of homogeneous functions $\mathcal{P}_{m}$ in the commutative case is replaced by the space of operators acting on the Fock space of three independent oscillators $\mathcal{F}_{N}^{(3)}$ (3.6). For instance, the monomial

$$
\frac{z_{0}^{m_{1}} z_{1}^{m_{2}} z_{2}^{m_{3}} \overline{z_{0}} m_{1}^{\prime} \bar{z}_{1}^{m_{2}^{\prime}} \overline{z_{2}} m_{3}^{\prime}}{\left(\left|z_{0}\right|^{2}+\left|z_{1}\right|^{2}+\left|z_{2}\right|^{2}\right)^{m}}, \quad\left(\sum_{i=1}^{3} m_{i}=\sum_{i=1}^{3} m_{i}^{\prime}=m\right)
$$

corresponds to the operator acting on $\mathcal{F}_{N}^{(3)}$

$$
\frac{1}{N^{m}}\left(a_{1}^{\dagger}\right)^{m_{1}^{\prime}}\left(a_{2}^{\dagger}\right)^{m_{2}^{\prime}}\left(a_{3}^{\dagger}\right)^{m_{3}^{\prime}}\left(a_{1}\right)^{m_{1}}\left(a_{2}\right)^{m_{2}}\left(a_{3}\right)^{m_{3}} .
$$

Here we have used the usual normal ordering prescription to go from the commutative expression (4.12) to the operator (4.13). The upper bound for the harmonics $m \leq N$

comes from the fact that the operator in (4.13) annihilates all states in $\mathcal{F}_{N}^{(3)}$ if $m$ exceeds $N$. We can also check that the total number of eigen-modes agrees with the dimension of the algebra $\mathbb{C P}_{N}^{2}=\operatorname{End}\left(\mathcal{F}_{N}^{(3)}\right)$ :

$$
\sum_{m=0}^{N} \mu_{m}=\sum_{m=0}^{N}(m+1)^{3}=\left\{\frac{1}{2}(N+1)(N+2)\right\}^{2}=\left(\operatorname{dim} \mathcal{F}_{N}^{(3)}\right)^{2}
$$

As discussed in [4], the truncation of the KK spectrum in the F(uzz) theory may have interesting consequences in the low energy physics.

\subsection{KK Reduction of the Gauge Fields}

The fluctuation of gauge fields around the fuzzy $\mathbb{C P}^{1}$ solution in the $A_{1}$ quiver theory was studied in [19,21]. Here we repeat similar analysis for fuzzy $\mathbb{C P}^{2}$. The KK mass spectrum of the gauge fields is obtained from the kinetic terms of $A_{i}$ and $B_{i}$ :

$$
\begin{aligned}
& \mathcal{L}=\sum_{i=1}^{3} \operatorname{Tr}\left(\left|D_{t} A_{i}\right|^{2}+\left|D_{t} B_{i}\right|^{2}\right), \\
& D_{t} A_{i}=\partial_{t} A_{i}+i\left(W_{t}^{1} A_{i}-A_{i} W_{t}^{2}\right), \\
& D_{t} B_{i}=\partial_{t} B_{i}+i\left(W_{t}^{2} B_{i}-B_{i} W_{t}^{3}\right),
\end{aligned}
$$


where $W_{t}^{1,2,3}$ denotes the 1-dimensional gauge field at the nodes 1, 2, and 3. From our interpretation of the solution as a D4-brane, we expect to obtain a single KK tower for a single gauge field living on the worldvolume of the single D4-brane, given by the eigenvalues of the scalar Laplacian on fuzzy $\mathbb{C P}^{2}(4.5)$. We will see below that this is indeed true for the low-lying excitations in the large $N$ limit.

Expanding (4.15) around the classical solution (3.14), the mass term for the gauge field becomes

$$
\begin{aligned}
\mathcal{L}_{\text {mass }} & =|c|^{2} \sum_{i=1}^{3} \operatorname{Tr}\left[a_{i} a_{i}^{\dagger}\left(W_{t}^{1}\right)^{2}+a_{i}^{\dagger} a_{i}\left(W_{t}^{2}\right)^{2}-a_{i}^{\dagger} W_{t}^{1} a_{i} W_{t}^{2}-W_{t}^{1} a_{i} W_{t}^{2} a_{i}^{\dagger}\right] \\
& +|\widetilde{c}|^{2} \sum_{i=1}^{3} \operatorname{Tr}\left[a_{i} a_{i}^{\dagger}\left(W_{t}^{2}\right)^{2}+a_{i}^{\dagger} a_{i}\left(W_{t}^{3}\right)^{2}-a_{i}^{\dagger} W_{t}^{2} a_{i} W_{t}^{3}-W_{t}^{2} a_{i} W_{t}^{3} a_{i}^{\dagger}\right] .
\end{aligned}
$$

As discussed in 19,21, since the eigenvalue is common for all scalar harmonics belonging to the same representation $[m, m]$ of $S U(3)$, it is sufficient to consider one particular mode

$$
\phi_{m}=\left(a_{1}^{\dagger} a_{2}\right)^{m}
$$

We expand the gauge fields as

$$
W_{t}^{r}=g \frac{W_{t}^{r, m} \cdot\left(\phi_{r, m}+\phi_{r, m}^{\dagger}\right)}{\sqrt{\operatorname{Tr}\left(\phi_{r, m}+\phi_{r, m}^{\dagger}\right)^{2}}}, \quad(r=1,2,3)
$$

where $\phi_{r, m}$ denotes the restriction of $\phi_{m}$ to the $r$-th Chan-Paton space $\mathbb{C}^{n_{r}}$ in (3.5), and $g$ denotes the gauge coupling. Here we have assumed for simplicity that the gauge couplings of all three gauge groups $U\left(n_{r}\right)$ have a common value $g$. The normalization factor $g / \sqrt{\operatorname{Tr}\left(\phi_{r, m}+\phi_{r, m}^{\dagger}\right)^{2}}$ in $(4.18)$ is included so that the kinetic term of $W_{t}^{r, m}$ becomes canonical [19].

The diagonal terms of the mass matrix in (4.16) are found by using (3.16). In order to evaluate the mixing terms in (4.16), we first notice the relation

$$
\begin{aligned}
& \sum_{i=1}^{3} a_{i}^{\dagger} \phi_{m} a_{i}=(\widehat{N}-m) \phi_{m} \\
& \sum_{i=1}^{3} a_{i} \phi_{m} a_{i}^{\dagger}=(\widehat{N}+3+m) \phi_{m}
\end{aligned}
$$


where $\widehat{N}=\sum_{i=1}^{3} a_{i}^{\dagger} a_{i}$ is the total number operator. From this relation, we obtain

$$
\begin{aligned}
& \sum_{i=1}^{3} a_{i}^{\dagger}\left(\phi_{1, m}+\phi_{1, m}^{\dagger}\right) a_{i}=(N+1-m)\left(\phi_{2, m}+\phi_{2, m}^{\dagger}\right) \\
& \sum_{i=1}^{3} a_{i}\left(\phi_{2, m}+\phi_{2, m}^{\dagger}\right) a_{i}^{\dagger}=(N+3+m)\left(\phi_{1, m}+\phi_{1, m}^{\dagger}\right) \\
& \sum_{i=1}^{3} a_{i}^{\dagger}\left(\phi_{2, m}+\phi_{2, m}^{\dagger}\right) a_{i}=(N+2-m)\left(\phi_{3, m}+\phi_{3, m}^{\dagger}\right) \\
& \sum_{i=1}^{3} a_{i}\left(\phi_{3, m}+\phi_{3, m}^{\dagger}\right) a_{i}^{\dagger}=(N+4+m)\left(\phi_{2, m}+\phi_{2, m}^{\dagger}\right) .
\end{aligned}
$$

Furthermore, by noticing that

$$
\begin{aligned}
& \sum_{i=1}^{3} \operatorname{Tr}\left(\phi_{1, m}+\phi_{1, m}^{\dagger}\right) a_{i}\left(\phi_{2, m}+\phi_{2, m}^{\dagger}\right) a_{i}^{\dagger}=(N+3+m) \operatorname{Tr}\left(\phi_{1, m}+\phi_{1, m}^{\dagger}\right)^{2} \\
= & \sum_{i=1}^{3} \operatorname{Tr} a_{i}^{\dagger}\left(\phi_{1, m}+\phi_{1, m}^{\dagger}\right) a_{i}\left(\phi_{2, m}+\phi_{2, m}^{\dagger}\right)=(N+1-m) \operatorname{Tr}\left(\phi_{2, m}+\phi_{2, m}^{\dagger}\right)^{2},
\end{aligned}
$$

we find the identity

$$
\begin{aligned}
& (N+3+m) \operatorname{Tr}\left(\phi_{1, m}+\phi_{1, m}^{\dagger}\right)^{2}=(N+1-m) \operatorname{Tr}\left(\phi_{2, m}+\phi_{2, m}^{\dagger}\right)^{2} \\
= & \sqrt{(N+3+m)(N+1-m) \operatorname{Tr}\left(\phi_{1, m}+\phi_{1, m}^{\dagger}\right)^{2} \operatorname{Tr}\left(\phi_{2, m}+\phi_{2, m}^{\dagger}\right)^{2}} .
\end{aligned}
$$

Similarly, the mixing term of $W_{t}^{2}$ and $W_{t}^{3}$ becomes

$$
\begin{aligned}
& \sum_{i=1}^{3} \operatorname{Tr} a_{i}^{\dagger}\left(\phi_{2, m}+\phi_{2, m}^{\dagger}\right) a_{i}\left(\phi_{3, m}+\phi_{3, m}^{\dagger}\right) \\
= & \sum_{i=1}^{3} \operatorname{Tr}\left(\phi_{2, m}+\phi_{2, m}^{\dagger}\right) a_{i}\left(\phi_{3, m}+\phi_{3, m}^{\dagger}\right) a_{i}^{\dagger} \\
= & \sqrt{(N+4+m)(N+2-m) \operatorname{Tr}\left(\phi_{2, m}+\phi_{2, m}^{\dagger}\right)^{2} \operatorname{Tr}\left(\phi_{3, m}+\phi_{3, m}^{\dagger}\right)^{2}} .
\end{aligned}
$$

Finally, we find that the mass matrix of $W_{t}^{1,2,3}$ in the representation $[m, m]$ is given by

$$
\begin{aligned}
& \mathcal{M}=|g c|^{2}\left(\begin{array}{ccc}
N+3 & -\sqrt{(N+3)(N+1)-m(m+2)} & 0 \\
-\sqrt{(N+3)(N+1)-m(m+2)} & N+1 & 0 \\
0 & 0 & 0
\end{array}\right) \\
& +|g \widetilde{c}|^{2}\left(\begin{array}{ccc}
0 & 0 & 0 \\
0 & N+4 & -\sqrt{(N+4)(N+2)-m(m+2)} \\
0 & -\sqrt{(N+4)(N+2)-m(m+2)} & N+2
\end{array}\right) .
\end{aligned}
$$


The KK spectrum is obtained by diagonalizing this matrix. The characteristic equation $\operatorname{det}(\lambda-\mathcal{M})=0$ for the mass matrix reads

$$
\begin{aligned}
& \lambda^{3}-\left\{|g c|^{2}(N+2)+|g \widetilde{c}|^{2}(N+3)\right\}\left\{2 \lambda^{2}+|g c|^{2}|g \widetilde{c}|^{2} m(m+2)\right\} \\
& +\lambda\left\{m(m+2)\left(|g c|^{4}+|g \widetilde{c}|^{4}\right)+2|g c|^{2}|g \widetilde{c}|^{2}\left(n_{1}+n_{2}+n_{3}\right)\right\}=0
\end{aligned}
$$

and the KK eigenvalues are given by the solution of this cubic equation. Writing the exact solution of this characteristic equation is not so illuminating, so we consider the approximate behavior of the small $m$ eigenvalues in the large $N$ regime. When $m \ll N$, the eigenvalues are found to be

$$
\begin{aligned}
\lambda_{m, 0} \sim & \frac{|g c|^{2}(N+2)+|g \widetilde{c}|^{2}(N+3)}{2\left(n_{1}+n_{2}+n_{3}\right)} m(m+2) \\
\lambda_{m, \pm} \sim|g c|^{2}(N+2)+|g \widetilde{c}|^{2}(N+3) & \\
& \pm \sqrt{\left\{|g c|^{2}(N+2)-|g \widetilde{c}|^{2}(N+3)\right\}^{2}+|g c|^{2}|g \widetilde{c}|^{2}(N+1)(N+4)} .
\end{aligned}
$$

We can see that $\lambda_{m, 0}$ corresponds to the $m$-th eigenvalue of the scalar Laplacian on $\mathbb{C P}^{2}$ (4.5). Therefore, the low-lying KK modes reproduce the known spectrum of the scalar Laplacian on $\mathbb{C P}^{2}$. By comparing the commutative expression $\lambda_{m}=4 M_{\mathrm{KK}}^{2} m(m+2)$ in (4.5) and the eigenvalue $\lambda_{m, 0}$ in (4.26), we find that the KK mass scale for the compactification on fuzzy $\mathbb{C P}^{2}$ is given by

$$
M_{\mathrm{KK}}^{2} \sim \frac{|g c|^{2}(N+2)+|g \widetilde{c}|^{2}(N+3)}{n_{1}+n_{2}+n_{3}} .
$$

This KK mass scale behaves as $M_{\mathrm{KK}}^{2} \sim N^{-1}$ when $N \gg 1$, which suggests that our fuzzy $\mathbb{C P}^{2}$ solution approaches a smooth large volume $\mathbb{C P}^{2}$ in the large $N$ limit. On the other hand, the interpretation of other eigenvalues $\lambda_{m, \pm}$ in (4.26) is not so clear. As discussed in [21], $\lambda_{m, \pm}$ may be related to a UV effect in the theory on fuzzy $\mathbb{C P}^{2}$. Note that $|g c|,|g \widetilde{c}|$ in (4.27) have the usual form of $\mathrm{W}$-boson mass since the gauge symmetry is completely Higgsed by the VEV of the scalar fields $\left\langle A_{i}\right\rangle=c a_{i},\left\langle B_{i}\right\rangle=\widetilde{c} a_{i}$.

The expression of the mass matrix in (4.24) is valid for $m=0, \cdots, N$. When $m=$ $N+1$, we have $\phi_{1, m}=0$ and hence we have to diagonalize the mass matrix for $W_{t}^{2,3}$. Similarly, for $m=N+2$ we have $\phi_{1, m}=\phi_{2, m}=0$, and the eigenvalue of $W_{t}^{3}$ is simply $|g \widetilde{c}|^{2}(N+2)$. 


\subsection{Mass Matrix for the Field $C_{i}$}

From the superpotential $W=\varepsilon^{i j k} \operatorname{Tr}\left(A_{i} B_{j} C_{k}\right)$, we obtain the potential for the field $C_{i}$ as follows:

$$
\begin{aligned}
V\left(C_{i}\right) & =\left|\frac{\partial W}{\partial A_{k}}\right|^{2}+\left|\frac{\partial W}{\partial B_{k}}\right|^{2} \\
= & \operatorname{Tr}_{\mathcal{F}_{N+2}}\left(C_{i} A_{j} A_{j}^{\dagger} C_{i}^{\dagger}-C_{i} A_{j} A_{i}^{\dagger} C_{j}^{\dagger}\right) \\
& +\operatorname{Tr}_{\mathcal{F}_{N}}\left(C_{i}^{\dagger} B_{j}^{\dagger} B_{j} C_{i}-C_{i}^{\dagger} B_{j}^{\dagger} B_{i} C_{j}\right) .
\end{aligned}
$$

Expanding around the fuzzy $\mathbb{C P}^{2}$ solution (3.14) up to the quadratic ordert, we obtain the mass term for the field $C_{i}$ :

$$
\begin{aligned}
& |c|^{2} \operatorname{Tr}_{\mathcal{F}_{N+2}}\left\{\left.C_{i}\left(\delta^{i i^{\prime}} \delta^{j j^{\prime}}-\delta^{i j^{\prime}} \delta^{j i^{\prime}}\right) a_{j} a_{j^{\prime}}^{\dagger}\right|_{\mathcal{F}_{N}} C_{i^{\prime}}^{\dagger}\right\} \\
& +|\bar{c}|^{2} \operatorname{Tr}_{\mathcal{F}_{N}}\left\{\left.C_{i^{\prime}}^{\dagger}\left(\delta^{i i^{\prime}} \delta^{j j^{\prime}}-\delta^{i^{\prime} j^{\prime}} \delta^{j i}\right) a_{j}^{\dagger} a_{j^{\prime}}\right|_{\mathcal{F}_{N+2}} C_{i}\right\} .
\end{aligned}
$$

In the first line of (4.29), we can rewrite as

$$
\begin{aligned}
\left.\left(\delta^{i i^{\prime}} \delta^{j j^{\prime}}-\delta^{i j^{\prime}} \delta^{j i^{\prime}}\right) a_{j} a_{j^{\prime}}^{\dagger}\right|_{\mathcal{F}_{N}} & =(N+3) \delta^{i i^{\prime}}-\left.a_{i^{\prime}} a_{i}^{\dagger}\right|_{\mathcal{F}_{N}} \\
& =\left.(N+2)\left(\delta^{i i^{\prime}}-\frac{a_{i}^{\dagger} a_{i^{\prime}}}{N+2}\right)\right|_{\mathcal{F}_{N}} .
\end{aligned}
$$

Similarly, in the second line of (4.29),

$$
\begin{aligned}
\left.\left(\delta^{i i^{\prime}} \delta^{j j^{\prime}}-\delta^{i^{\prime} j^{\prime}} \delta^{j i}\right) a_{j}^{\dagger} a_{j^{\prime}}\right|_{\mathcal{F}_{N+2}} & =\delta^{i i^{\prime}}(N+2)-\left.a_{i}^{\dagger} a_{i^{\prime}}\right|_{\mathcal{F}_{N+2}} \\
& =\left.\delta^{i i^{\prime}}(N+3)\left(\delta^{i i^{\prime}}-\frac{a_{i^{\prime}} a_{i}^{\dagger}}{N+3}\right)\right|_{\mathcal{F}_{N+2}} .
\end{aligned}
$$

We may write the mass term (4.29) in the following form:

$$
C_{\vec{n}_{1} \vec{n}_{2}}^{i} \mathcal{M}_{\left(\vec{n}_{1} \vec{n}_{2}\right)\left(\vec{n}_{3} \vec{n}_{4}\right)}^{i j} C_{\vec{n}_{3} \vec{n}_{4}}^{\dagger j}
$$

where we have introduced a shorthand notation

$$
|\vec{n}\rangle=\left|n_{1}, n_{2}, n_{3}\right\rangle, \quad\langle\vec{n}|=\left\langle n_{1}, n_{2}, n_{3}\right|,
$$

7 The D-term potential does not contain the term quadratic in the fluctuation of the filed $C_{i}$ around the classical solution (3.14). 
and

$$
C^{i}=C_{\vec{n}_{1} \vec{n}_{2}}^{i}\left|\vec{n}_{1}\right\rangle\left\langle\vec{n}_{2}\right| \text {. }
$$

In (4.32), $\left|\vec{n}_{1}\right\rangle,\left|\vec{n}_{4}\right\rangle \in \mathcal{F}_{N+2}$ and $\left|\vec{n}_{2}\right\rangle,\left|\vec{n}_{3}\right\rangle \in \mathcal{F}_{N}$. Using (4.30) and (4.31), the mass matrix $\mathcal{M}$ is obtained as

$$
\begin{aligned}
\mathcal{M}_{\left(\vec{n}_{1} \vec{n}_{2}\right)\left(\vec{n}_{3} \vec{n}_{4}\right)}^{i j}= & |c|^{2}(N+2)\left(1-P_{A}\right)_{\left(\vec{n}_{1} \vec{n}_{2}\right)\left(\vec{n}_{3} \vec{n}_{4}\right)}^{i j} \\
& +|\widetilde{c}|^{2}(N+3)\left(1-P_{B}\right)_{\left(\vec{n}_{1} \vec{n}_{2}\right)\left(\vec{n}_{3} \vec{n}_{4}\right)}^{i j},
\end{aligned}
$$

where

$$
\begin{aligned}
& P_{A\left(\vec{n}_{1} \vec{n}_{2}\right)\left(\vec{n}_{3} \vec{n}_{4}\right)}^{i j} \equiv\left(\left.\frac{a_{i}^{\dagger} a_{j}}{N+2}\right|_{\mathcal{F}_{N}}\right)_{\vec{n}_{2} \vec{n}_{3}}\left(\mathbf{1}_{\mathcal{F}_{N+2}}\right)_{\vec{n}_{1} \vec{n}_{4}} \\
& P_{B\left(\vec{n}_{1} \vec{n}_{2}\right)\left(\vec{n}_{3} \vec{n}_{4}\right)}^{i j} \equiv\left(\mathbf{1}_{\mathcal{F}_{N}}\right)_{\vec{n}_{2} \vec{n}_{3}}\left(\left.\frac{a_{j} a_{i}^{\dagger}}{N+3}\right|_{\mathcal{F}_{N+2}}\right)_{\vec{n}_{4} \vec{n}_{1}},
\end{aligned}
$$

are projections. Dimensions of these projections are given by

$$
\begin{aligned}
\operatorname{dim} P_{A}=\operatorname{Tr} P_{A} & =\operatorname{Tr}_{\mathcal{F}_{N}} \frac{a_{i}^{\dagger} a_{i}}{N+2} \operatorname{Tr}_{\mathcal{F}_{N+2}} \mathbf{1} \\
& =\frac{N}{N+2} \operatorname{dim} \mathcal{F}_{N} \operatorname{dim} \mathcal{F}_{N+2} \\
& =\operatorname{dim} \mathcal{F}_{N-1} \mathcal{F}_{N+2},
\end{aligned}
$$

(here we assume $N \geq 2$ ), and

$$
\begin{aligned}
\operatorname{dim} P_{B}=\operatorname{Tr} P_{B} & =\operatorname{Tr}_{\mathcal{F}_{N}} \mathbf{1} \operatorname{Tr}_{\mathcal{F}_{N+2}} \frac{a_{i} a_{i}^{\dagger}}{N+3} \\
& =\operatorname{dim} \mathcal{F}_{N} \operatorname{dim} \mathcal{F}_{N+2} \frac{N+5}{N+3} \\
& =\operatorname{dim} \mathcal{F}_{N} \operatorname{dim} \mathcal{F}_{N+3} .
\end{aligned}
$$

We can explicitly construct the basis of the projected space for each projection. For the projection $P_{A}$, they are given by

$$
\left(a_{j}^{\dagger}\right)_{\vec{n}_{3} \vec{n}} C_{\vec{n} \vec{n}_{4}}^{A}
$$

where $\left|\vec{n}_{3}\right\rangle \in \mathcal{F}_{N},|\vec{n}\rangle \in \mathcal{F}_{N-1},\left|\vec{n}_{4}\right\rangle \in \mathcal{F}_{N+2} . C_{\vec{n} \vec{n}_{2}}^{A}$ is a $\operatorname{dim} \mathcal{F}_{N-1} \times \operatorname{dim} \mathcal{F}_{N+2}$ complex matrix, so there are $\operatorname{dim} \mathcal{F}_{N-1} \times \operatorname{dim} \mathcal{F}_{N+2}$ independent such matrices, in agreement with (4.38).8 For the projection $P_{B}$, those are given by

$$
C_{\vec{n}_{3} \vec{n}^{\prime}}^{B \dagger}\left(a_{j}^{\dagger}\right)_{\vec{n}^{\prime} \vec{n}_{4}}
$$

8 The mass term (4.32) has a natural Hermitian structure. 
where $\left|\vec{n}_{3}\right\rangle \in \mathcal{F}_{N},\left|\vec{n}^{\prime}\right\rangle \in \mathcal{F}_{N+3},\left|\vec{n}_{4}\right\rangle \in \mathcal{F}_{N+2} . \quad C_{\vec{n}_{3} \vec{n}^{\prime}}^{B \dagger}$ is a $\operatorname{dim} \mathcal{F}_{N} \times \operatorname{dim} \mathcal{F}_{N+3}$ complex matrix, so there are $\operatorname{dim} \mathcal{F}_{N} \times \operatorname{dim} \mathcal{F}_{N+3}$ independent such matrices, in agreement with (4.39).

When $\widetilde{c}=0$ (or $c=0$, respectively below), the mass is $|c|^{2}(N+2)\left(|\widetilde{c}|^{2}(N+3)\right)$ for the components of $C_{i}$ projected by $1-P_{A}\left(1-P_{B}\right)$, and zero for those projected by $P_{A}$ $\left(P_{B}\right)$. However, since two projections $P_{A}$ and $P_{B}$ do not commute with each other i.e. $\left[P_{A}, P_{B}\right] \neq 0$, they cannot be simultaneously diagonalizable. Therefore, diagonalization of the mass matrix is a rather non-trivial problem when $c \widetilde{c} \neq 0$. We leave this problem to the future.

\section{Fuzzy del Pezzo Surfaces from Quiver Gauge Theories}

In this section we will construct classical solutions of the quiver gauge theories on the D0-branes probing del Pezzo singularities. Our solutions describe D4-branes wrapped on fuzzy $d P_{k}$ for $k=1,2,3$.

\subsection{Fuzzy $d P_{1}$}

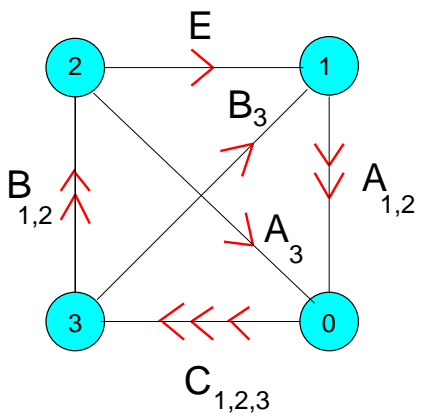

(a)

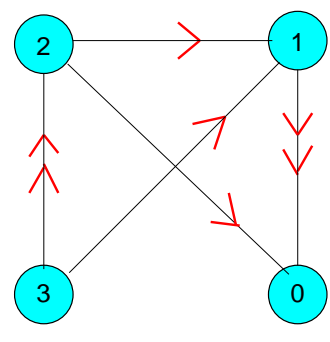

(b)

Fig. 3: (a) The quiver diagram for the worldline theory of D0-branes probing a $d P_{1}$ singularity. (b) A classical solution describing D4-branes wrapped on fuzzy $d P_{1}$ can be obtained by setting $C_{i}=0$. This corresponds to deleting the arrows between the node 0 and the node 3 in the diagram (a).

Let us first construct a classical solution of quiver gauge theory for the D0-branes probing the $d P_{1}$ singularity. The quiver diagram of this theory is depicted in Fig. 3a. The superpotential of this quiver gauge theory is given by [32, 33, 34, 35.

$$
W=\operatorname{Tr}\left[A_{1} E B_{2} C_{3}-A_{2} E B_{1} C_{3}+A_{2} B_{3} C_{1}-A_{3} B_{2} C_{1}+A_{3} B_{1} C_{2}-A_{1} B_{3} C_{2}\right] .
$$


We consider a classical solution with $C_{i}=0(i=1,2,3)$. This corresponds to deleting the arrows between the node 0 and the node 3 (see Fig. 3b9). After deleting those arrows, the remaining non-zero fields are $A_{i}, B_{i}(i=1,2,3$,$) and E$. When $C_{i}=0$ the F-term condition for the other non-zero fields reads

$$
A_{1} E B_{2}=A_{2} E B_{1}, \quad A_{2} B_{3}=A_{3} B_{2}, \quad A_{3} B_{1}=A_{1} B_{3}
$$

The D-term condition is

$$
\begin{aligned}
& \zeta_{0} \mathbf{1}_{V_{0}}=A_{1} A_{1}^{\dagger}+A_{2} A_{2}^{\dagger}+A_{3} A_{3}^{\dagger} \\
& \zeta_{1} \mathbf{1}_{V_{1}}=E E^{\dagger}+B_{3} B_{3}^{\dagger}-A_{1}^{\dagger} A_{1}-A_{2}^{\dagger} A_{2} \\
& \zeta_{2} \mathbf{1}_{V_{2}}=B_{1} B_{1}^{\dagger}+B_{2} B_{2}^{\dagger}-E^{\dagger} E-A_{3}^{\dagger} A_{3} \\
& \zeta_{3} \mathbf{1}_{V_{3}}=-B_{1}^{\dagger} B_{1}-B_{2}^{\dagger} B_{2}-B_{3}^{\dagger} B_{3} .
\end{aligned}
$$

One obvious solution to the F-term (5.2) and the D-term conditions (5.3) is obtained by setting

$$
A_{i}=c a_{i}, \quad B_{i}=\widetilde{c} a_{i} \quad(i=1,2,3), \quad E=\sqrt{|c|^{2}+|\widetilde{c}|^{2}} a_{4}
$$

where $a_{i}$ 's are four independent oscillators and $c, \widetilde{c}$ are complex numbers. In order to satisfy the D-term condition (5.3), we take the Chan-Paton spaces as

$$
V_{3}=\mathcal{F}_{N+2, M+1}, \quad V_{2}=\mathcal{F}_{N+1, M+1}, \quad V_{1}=\mathcal{F}_{N+1, M}, \quad V_{0}=\mathcal{F}_{N, M},
$$

where $V_{r}(r=0,1,2,3)$ represents the Chan-Paton space at the node $r$ and the space $\mathcal{F}_{N, M}$ is defined by

$$
\mathcal{F}_{N, M}=\left\{\left|m_{1}, m_{2}, m_{3}, m_{4}\right\rangle, \quad \sum_{i=1}^{3} m_{i}=N, \quad m_{3}+m_{4}=M\right\} .
$$

Then the FI parameter is quantized as

$$
\begin{aligned}
& \zeta_{0}=|c|^{2}(N+3) \\
& \zeta_{1}=-|c|^{2}(N-M)+|\widetilde{c}|^{2}(M+2) \\
& \zeta_{2}=-|c|^{2}(M+1)+|\widetilde{c}|^{2}(N-M+2) \\
& \zeta_{3}=-|\widetilde{c}|^{2}(N+2) .
\end{aligned}
$$

9 This is different from the Beilinson quiver of $d P_{1}$ considered in [36], eq.(33). However, as discussed around eq.(66) in [36], we can transform the Beilinson quiver of $d P_{1}$ in [36] into our Beilinson quiver in Fig. $3 b$ by a series of mutations.

For further discussions on the Beilinson quiver in related contexts, see [37,38, 39, 40, 41]. 
The dimension of the space $\mathcal{F}_{N, M}$ can be counted from the quantized toric diagram of the fuzzy $d P_{1}$ (Fig. 4). It is given by the total number of the black points:

$$
\begin{aligned}
\operatorname{dim} \mathcal{F}_{N, M} & =\frac{1}{2}(N+1)(N+2)-\frac{1}{2}(N-M)(N-M+1) \\
& =\frac{1}{2}(M+1)(2 N+2-M) .
\end{aligned}
$$

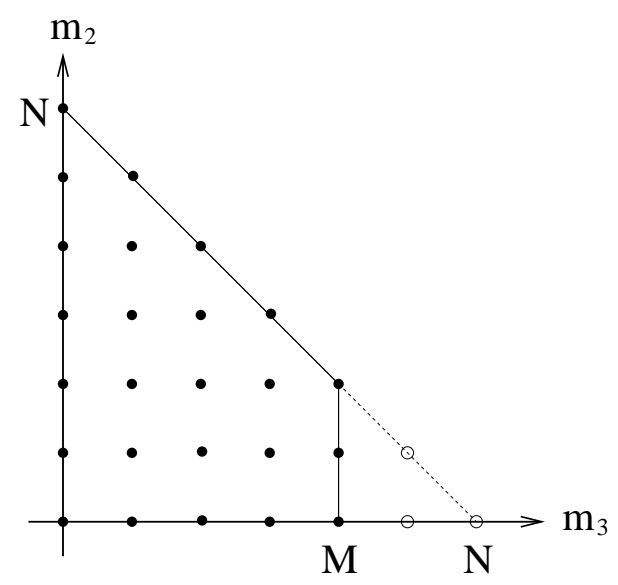

Fig. 4: "Quantized" toric diagram of fuzzy $d P_{1}$. The black points are the points in the base space of fuzzy $d P_{1}$ when it is looked as a torus fibered over the base space. Compared with the toric diagram of fuzzy $\mathbb{C P}^{2}$ Fig. 2, white points in this figure are removed, which is regarded as a fuzzy version of the blow-up at a point $z_{1}=z_{2}=0$.

Using (5.8), one can show that

$$
\sum_{r=0}^{3} \zeta_{r} \operatorname{dim} V_{r}=0,
$$

which means that the overall $U(1)$ subgroup of the gauge group is decoupled. Let us represent the $U(1)$ subgroup of the $U\left(n_{r}\right)$ gauge group at the node $r$ by $e^{i \theta_{r}}(r=0,1,2,3)$. Since the overall $U(1)$ phase decouples, we have three $U(1)$ phases left. We have gauge equivalence relations

$$
\begin{aligned}
A_{1,2} & \sim e^{i\left(\theta_{1}-\theta_{0}\right)} A_{1,2}, \quad A_{3} \sim e^{i\left(\theta_{2}-\theta_{0}\right)} A_{3}=e^{i\left(\left(\theta_{2}-\theta_{1}\right)+\left(\theta_{1}-\theta_{0}\right)\right)} A_{3}, \\
B_{1,2} & \sim e^{i\left(\theta_{3}-\theta_{2}\right)} B_{1,2}, \quad B_{3} \sim e^{i\left(\theta_{3}-\theta_{1}\right)} B_{3}=e^{i\left(\left(\theta_{3}-\theta_{2}\right)+\left(\theta_{2}-\theta_{1}\right)\right)} B_{3}, \\
E & \sim e^{i\left(\theta_{2}-\theta_{1}\right)} E,
\end{aligned}
$$

thus we can choose $\theta_{1}-\theta_{0}, \theta_{3}-\theta_{2}, \theta_{2}-\theta_{1}$ as three independent $U(1)$ phases. We observe that (5.10] corresponds to eq. (33) of [25], while (5.6) corresponds to eq.(32) of [25]. On 
the other hand, $m_{1}+m_{2}+m_{3}=N$ and $m_{3}+m_{4}=M$ in (5.6) corresponds to eq.(32) of [25]. Thus, our classical solution (5.4) can be regarded as a fuzzy version of the $d P_{1}$ surface. Let us have a closer look on our fuzzy $d P_{1}$. Recall that the toric diagram of $\mathbb{C P}^{2}$ (Fig. 2). Since blowing up at a point on $\mathbb{C P}^{2}$ corresponds to replacing a vertex of the triangle by a line segment, the blowing-up procedure in the fuzzy setup can be realized by removing some of the states in the Fock space $\mathcal{F}_{N}^{(3)}$. This is indeed the case for our space $\mathcal{F}_{N, M}=\mathcal{F}_{N, M}\left(d P_{1}\right)$. If we suppress the dependence on the fourth oscillator, the condition $m_{3}+m_{4}=M$ implies the upper bound on $m_{3}$ :

$$
\mathcal{F}_{N, M}\left(d P_{1}\right)=\left\{\left|m_{1}, m_{2}, m_{3}\right\rangle, \quad \sum_{i=1}^{3} m_{i}=N, \quad m_{3} \leq M\right\} .
$$

Hence the states with $m_{3}>M$ are removed from $\mathcal{F}_{N}^{(3)}$ as indicated by by the white points in Fig. 4. The vertex at $|0,0, N\rangle$ is replaced by fuzzy $\mathbb{C P}^{1}$

$$
\mathcal{F}_{N-M}\left(\mathbb{C P}^{1}\right)=\left\{\left|m_{1}, m_{2}, M\right\rangle, m_{1}+m_{2}=N-M\right\}
$$

which corresponds to the exceptional curve appeared in the blow-up of $\mathbb{C P}^{2}$ at a point.

As discussed in [4], by sandwiching between the projection operator

$$
\mathcal{P}_{d P_{1}}: \mathcal{F}_{N}^{(3)}=\mathcal{F}_{N}\left(\mathbb{C P}^{2}\right) \rightarrow \mathcal{F}_{N, M}\left(d P_{1}\right)
$$

we can compute various quantities such as Yukawa couplings on fuzzy $d P_{1}$ from the knowledge of the parent fuzzy $\mathbb{C P}^{2}$.

\subsection{Fuzzy $d P_{2}$}

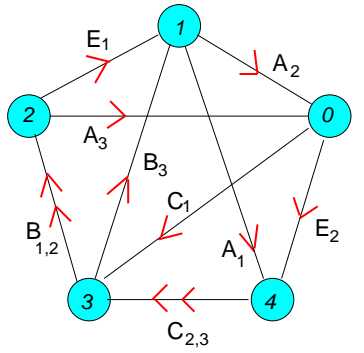

(a)

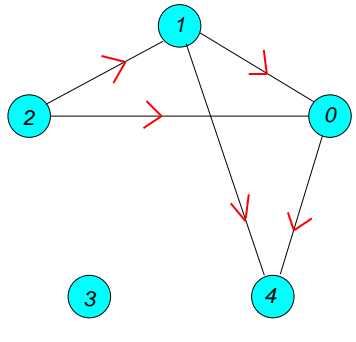

(b)

Fig. 5: (a) The quiver diagram for the worldline theory of D0-branes probing the $d P_{2}$ singularity. (b) A classical solution describing D4-branes wrapped on fuzzy $d P_{2}$ can be obtained by setting $B_{i}=C_{i}=0$. 
We can construct a classical solution of the $d P_{2}$ quiver gauge theory in a similar manner as in the $d P_{1}$ case in the previous subsection. The quiver diagram for the $d P_{2}$ singularity is depicted in Fig. 5a. The superpotential is given by [32, 33, 34, 35]

$$
W=\operatorname{Tr}\left[A_{1} E_{1} B_{2} C_{3}-A_{2} E_{1} B_{1} C_{3} E_{2}+A_{2} B_{3} C_{1}-A_{3} B_{2} C_{1}+A_{3} B_{1} C_{2} E_{2}-A_{1} B_{3} C_{2}\right]
$$

Contrary to the case of $d P_{1}$, if we set only the fields $C_{i}$ 's to zero it is not easy to satisfy the F-term condition coming from the superpotential (5.14) with simultaneously satisfying the D-term condition. Therefore, we set $B_{i}=C_{i}=0$ to find a classical solution for fuzzy $d P_{2}$, which corresponds to the diagram in Fig. 5b. Then the F-term condition is automatically satisfied. The D-term condition for the remaining non-zero fields $A_{1,2,3}, E_{1,2}$ reads

$$
\begin{aligned}
& \zeta_{4} \mathbf{1}_{V_{4}}=A_{1} A_{1}^{\dagger}+E_{2} E_{2}^{\dagger} \\
& \zeta_{0} \mathbf{1}_{V_{0}}=A_{2} A_{2}^{\dagger}+A_{3} A_{3}^{\dagger}-E_{2}^{\dagger} E_{2} \\
& \zeta_{1} \mathbf{1}_{V_{1}}=E_{1} E_{1}^{\dagger}-A_{1}^{\dagger} A_{1}-A_{2}^{\dagger} A_{2} \\
& \zeta_{2} \mathbf{1}_{V_{2}}=-E_{1}^{\dagger} E_{1}-A_{3}^{\dagger} A_{3} .
\end{aligned}
$$

This set of equations is solved by

$$
A_{i}=c a_{i}, \quad E_{1}=c a_{4}, \quad E_{2}=c a_{5},
$$

where $a_{i}(i=1, \ldots, 5)$ are five independent oscillators and the coefficient $c$ is a complex number. We choose the Chan-Paton spaces as

$$
V_{4}=\mathcal{F}_{N, M_{1}, M_{2}}, \quad V_{0}=\mathcal{F}_{N, M_{1}, M_{2}+1}, \quad V_{1}=\mathcal{F}_{N+1, M_{1}, M_{2}+1}, \quad V_{2}=\mathcal{F}_{N+1, M_{1}+1, M_{2}+1},
$$

where $\mathcal{F}_{N, M_{1}, M_{2}}$ is defined as

$$
\mathcal{F}_{N, M_{1}, M_{2}}=\left\{\left|m_{1}, m_{2}, m_{3}, m_{4}, m_{5}\right\rangle, \quad \sum_{i=1}^{3} m_{i}=N, \quad m_{3}+m_{4}=M_{1}, \quad m_{1}+m_{5}=M_{2}\right\} .
$$

The dimension of the space $\mathcal{F}_{N, M_{1}, M_{2}}$ can be calculated from Fig. 6 and is given by

$\operatorname{dim} \mathcal{F}_{N, M_{1}, M_{2}}=\frac{1}{2}(N+1)(N+2)-\frac{1}{2}\left(N-M_{1}\right)\left(N-M_{1}+1\right)-\frac{1}{2}\left(N-M_{2}\right)\left(N-M_{2}+1\right)$. 


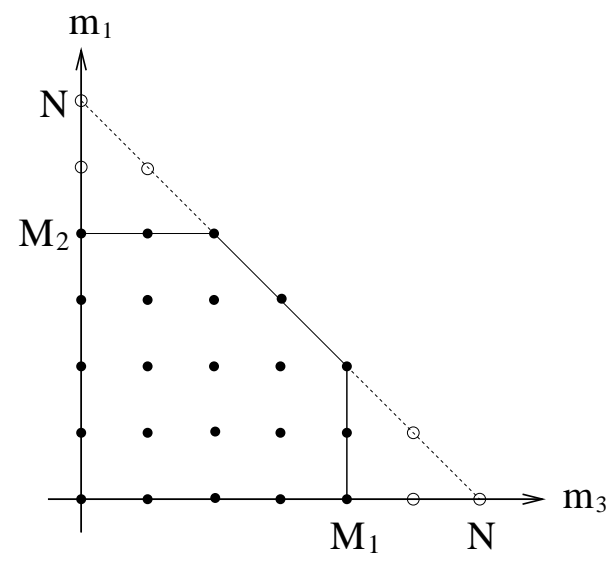

Fig. 6: "Quantized" toric diagram of fuzzy $d P_{2}$.

The FI parameters are quantized as follows

$$
\begin{aligned}
& \zeta_{4}=|c|^{2}\left(M_{2}+2\right) \\
& \zeta_{0}=|c|^{2}\left(N-M_{2}+1\right) \\
& \zeta_{1}=-|c|^{2}\left(N-M_{1}\right) \\
& \zeta_{2}=-|c|^{2}\left(M_{1}+1\right) .
\end{aligned}
$$

Using (5.19) one can show that

$$
\sum_{r=0,1,2,4} \zeta_{r} \operatorname{dim} V_{r}=0
$$

which means that the overall $U(1)$ of the gauge group is decoupled. Let us represent the $U(1)$ subgroup at the node $r$ by $e^{i \theta_{r}}(r=0,1,2,4)$. Since the overall $U(1)$ phase decouples, we have three $U(1)$ phases left. We have gauge equivalent relations

$$
\begin{aligned}
& A_{1} \sim e^{i\left(\theta_{1}-\theta_{4}\right)} A_{1}=e^{i\left(\left(\theta_{1}-\theta_{0}\right)+\left(\theta_{0}-\theta_{4}\right)\right)} A_{1} \\
& A_{2} \sim e^{i\left(\theta_{1}-\theta_{0}\right)} A_{2} \\
& A_{3} \sim e^{i\left(\theta_{2}-\theta_{0}\right)} A_{3}=e^{i\left(\left(\theta_{2}-\theta_{1}\right)+\left(\theta_{1}-\theta_{0}\right)\right)} A_{3} \\
& E_{1} \sim e^{i\left(\theta_{2}-\theta_{1}\right)} E_{1} \\
& E_{2} \sim e^{i\left(\theta_{0}-\theta_{4}\right)} E_{2},
\end{aligned}
$$

thus we can choose $\theta_{1}-\theta_{0}, \theta_{2}-\theta_{1}, \theta_{0}-\theta_{4}$ as three independent $U(1)$ phases. We observe that (5.22) corresponds to eq.(37) of [25], while (5.18) corresponds to eq.(36) of [25]. Thus 
our classical solution (5.16) can be regarded as a fuzzy version of the $d P_{2}$ surface. As in the case of fuzzy $d P_{1}$, the correspondence of the space $\mathcal{F}_{N, M_{1}, M_{2}}$ and the toric diagram of $d P_{2}$ Fig. 6 can be seen by forgetting the dependence on $m_{4}, m_{5}$ :

$$
\mathcal{F}_{N, M_{1}, M_{2}}\left(d P_{2}\right)=\left\{\left|m_{1}, m_{2}, m_{3}\right\rangle, \quad \sum_{i=1}^{3} m_{i}=N, \quad m_{3} \leq M_{1}, \quad m_{1} \leq M_{2}\right\} .
$$

The upper bounds on $m_{3}, m_{1}$ mean that the vertices at $|0,0, N\rangle$ and $|N, 0,0\rangle$ are replaced by line segments representing two fuzzy $\mathbb{C P}^{1}$ 's, which appeared as exceptional cycles in the blowing up of $\mathbb{C P}^{2}$ at two points.

As depicted in Fig. 7, there is another quiver gauge theory for the $d P_{2}$ singularity related to the quiver in Fig. 5 by Seiberg duality (or toric duality) [32,42,33, 43, For this quiver gauge theory, we can also construct a classical solution describing a D4-brane wrapped on fuzzy $d P_{2}$ by deleting some of the arrows in Fig 7a. As in the case of Fig. 5, the F-term condition is automatically satisfied for the configuration in Fig. 7b. The D-term condition reads

$$
\begin{aligned}
& \zeta_{0} \mathbf{1}_{V_{0}}=A_{1} A_{1}^{\dagger}+E_{2} E_{2}^{\dagger} \\
& \zeta_{1} \mathbf{1}_{V_{1}}=A_{2} A_{2}^{\dagger}-E_{1}^{\dagger} E_{1}-E_{2}^{\dagger} E_{2} \\
& \zeta_{2} \mathbf{1}_{V_{2}}=A_{3} A_{3}^{\dagger}+E_{1} E_{1}^{\dagger} \\
& \zeta_{3} \mathbf{1}_{V_{3}}=-\left(A_{1}^{\dagger} A_{1}+A_{2}^{\dagger} A_{2}+A_{3}^{\dagger} A_{3}\right) .
\end{aligned}
$$

The solution to these equations is again given by five independent oscillators in (5.16), and we choose the Chan-Paton spaces as

$$
V_{0}=\mathcal{F}_{N, M_{1}+1, M_{2}}, \quad V_{1}=\mathcal{F}_{N, M_{1}+1, M_{2}+1}, \quad V_{2}=\mathcal{F}_{N, M_{1}, M_{2}+1}, \quad V_{3}=\mathcal{F}_{N+1, M_{1}+1, M_{2}+1}
$$

Then the FI-parameters are quantized as follows

$$
\begin{aligned}
& \zeta_{0}=|c|^{2}\left(M_{2}+2\right) \\
& \zeta_{1}=|c|^{2}\left(N-M_{1}-M_{2}-1\right) \\
& \zeta_{2}=|c|^{2}\left(M_{1}+2\right) \\
& \zeta_{3}=-|c|^{2}(N+1) .
\end{aligned}
$$

Again, one can show that

$$
\sum_{r=0}^{3} \zeta_{r} \operatorname{dim} V_{r}=0
$$

The analysis of the $U(1)$ phases is similar to the previous examples and we will not repeat it here. 


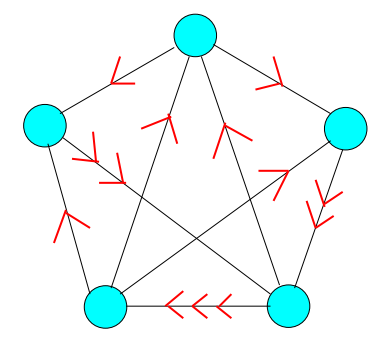

(a)

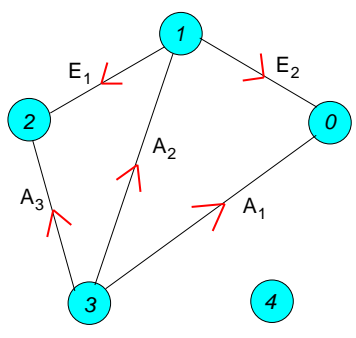

(b)

Fig. 7: (a) Another quiver diagram for the worldline theory of D0-branes probing the $d P_{2}$ singularity. This is related to the quiver in Fig. 5 by Seiberg duality. (b) A classical solution for the D-branes wrapped on fuzzy $d P_{2}$ can be obtained by deleting some arrows in the diagram (a).

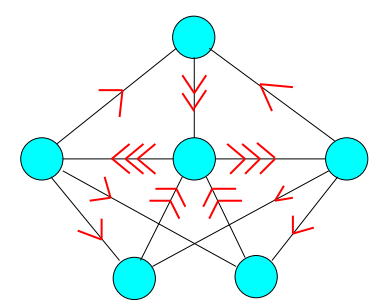

(a)

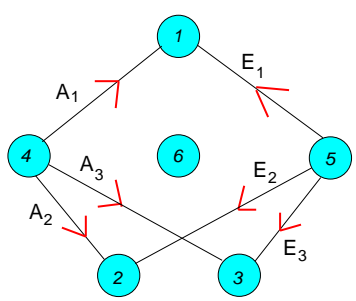

(b)

Fig. 8: (a) The quiver diagram for the worldline theory of D0-branes probing the $d P_{3}$ singularity. (b) A classical solution for the D-branes wrapped on fuzzy $d P_{3}$ can be obtained by deleting some arrows in the diagram (a).

\subsection{Fuzzy $d P_{3}$}

In a manner similar to the previous cases, we can construct a classical solution describing a D4-brane wrapped around fuzzy $d P_{3}$. Let us consider the quiver diagram for the $d P_{3}$ singularity depicted in Fig. 8a, which is called model IV in [33,44, 34]. A classical solution is obtained by deleting all arrows connected to the node 6 (see Fig. $8 \mathrm{~b}$ ). We take the remaining non-zero fields as the six independent oscillators

$$
A_{i}=c a_{i}, \quad E_{i}=c a_{i+3} \quad(i=1,2,3) .
$$

For this configuration the F-term condition is satisfied automatically, and the D-term condition reads

$$
\begin{aligned}
\zeta_{i} \mathbf{1}_{V_{i}} & =A_{i} A_{i}^{\dagger}+E_{i} E_{i}^{\dagger} \quad(i=1,2,3) \\
\zeta_{4} \mathbf{1}_{V_{4}} & =-\left(A_{1}^{\dagger} A_{1}+A_{2}^{\dagger} A_{2}+A_{3}^{\dagger} A_{3}\right) \\
\zeta_{5} \mathbf{1}_{V_{5}} & =-\left(E_{1}^{\dagger} E_{1}+E_{2}^{\dagger} E_{2}+E_{3}^{\dagger} E_{3}\right) .
\end{aligned}
$$


This is satisfied by taking the Chan-Paton spaces as

$$
\begin{aligned}
& V_{1}=\mathcal{F}_{N, M_{1}, M_{2}+1, M_{3}+1}, \quad V_{2}=\mathcal{F}_{N, M_{1}+1, M_{2}, M_{3}+1}, \quad V_{3}=\mathcal{F}_{N, M_{1}+1, M_{2}+1, M_{3}}, \\
& V_{4}=\mathcal{F}_{N+1, M_{1}+1, M_{2}+1, M_{3}+1}, \quad V_{5}=\mathcal{F}_{N, M_{1}+1, M_{2}+1, M_{3}+1},
\end{aligned}
$$

where

$$
\mathcal{F}_{N, M_{1}, M_{2}, M_{3}}=\left\{\prod_{k=1}^{6} \frac{\left(a_{k}^{\dagger}\right)^{m_{k}}}{\sqrt{m_{k} !}}|0\rangle, \quad \sum_{i=1}^{3} m_{i}=N, \quad m_{i}+m_{i+3}=M_{i} \quad(i=1,2,3)\right\} .
$$

The dimension of the space $\mathcal{F}_{N, M_{1}, M_{2}, M_{3}}$ can be calculated from Fig. 9 and is given by

$$
\begin{aligned}
\operatorname{dim} \mathcal{F}_{N, M_{1}, M_{2}}= & \frac{1}{2}(N+1)(N+2)-\frac{1}{2}\left(N-M_{1}\right)\left(N-M_{1}+1\right) \\
& -\frac{1}{2}\left(N-M_{2}\right)\left(N-M_{2}+1\right)-\frac{1}{2}\left(N-M_{3}\right)\left(N-M_{3}+1\right) .
\end{aligned}
$$

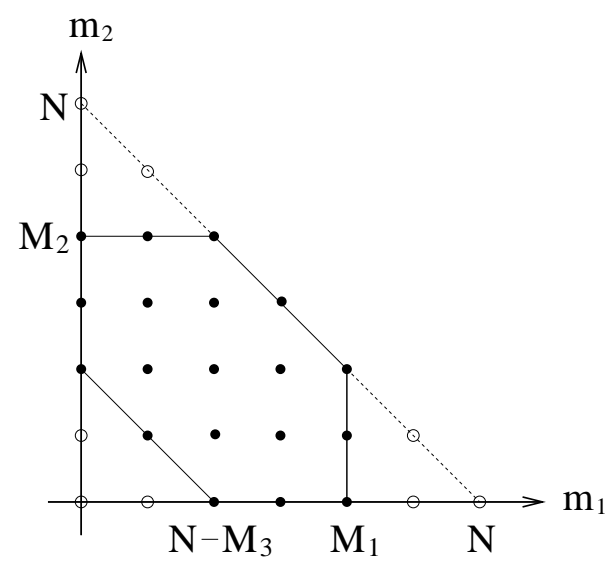

Fig. 9: "Quantized" toric diagram of fuzzy $d P_{3}$.

The FI-parameters are quantized as

$$
\begin{aligned}
& \zeta_{r}=|c|^{2}\left(M_{i}+2\right) \quad(r=1,2,3) \\
& \zeta_{4}=-|c|^{2}(N+1) \\
& \zeta_{5}=|c|^{2}\left(N-M_{1}-M_{2}-M_{3}-3\right) .
\end{aligned}
$$

One can show that

$$
\sum_{r=1}^{5} \zeta_{r} \operatorname{dim} V_{r}=0
$$


which means that the overall $U(1)$ of the gauge group is decoupled. Let us represent the $U(1)$ subgroup of $U\left(n_{r}\right)$ gauge group at the node $r$ by $e^{i \theta_{r}}(r=1,2,3,4,5)$. Since the overall $U(1)$ phase decouples, we have four $U(1)$ phases left. We have gauge equivalent relations

$$
\begin{aligned}
& A_{i} \sim e^{i\left(\theta_{4}-\theta_{i}\right)} A_{i}=e^{i\left(\left(\theta_{4}-\theta_{5}\right)+\left(\theta_{5}-\theta_{i}\right)\right)} A_{i} \quad(i=1,2,3) \\
& E_{i} \sim e^{i\left(\theta_{5}-\theta_{i}\right)} E_{i} .
\end{aligned}
$$

As before, these equivalence relations through the phases (5.35) together with (5.31) provides the toric description of $d P_{3}$. By forgetting the dependence of $m_{4,5,6}$, we have

$$
\mathcal{F}_{N, M_{1}, M_{2}, M_{3}}=\left\{\left|m_{1}, m_{2}, m_{3}\right\rangle, \quad \sum_{i=1}^{3} m_{i}=N, \quad m_{i} \leq M_{i}\right\} .
$$

We observe that the three vertices at $|0,0, N\rangle,|0, N, 0\rangle,|N, 0,0\rangle$ are replaced by line segments representing three fuzzy $\mathbb{C P}^{1}$ 's, which appeared as exceptional curves in the blowing up of $\mathbb{C P}^{2}$ at three points.

Some comments on our solution for the $d P_{2}$ and $d P_{3}$ cases are in order. In the case of $d P_{0}$ and $d P_{1}$, the gauge group is Higgsed completely around the classical solution if we set the parameters $c$ and $\widetilde{c}$ both non-zero. On the other hand, for the $d P_{2}$ and $d P_{3}$ cases our classical solutions have only one parameter $c$, and one of the gauge group remains unbroken around the classical solutions since the corresponding node is not connected to the other nodes in the quiver diagram for our solution. Our solutions for $d P_{2}$ and $d P_{3}$ are probably a class of simple solutions among more general solutions for the F-term condition, similar to $c=0$ (or $\tilde{c}=0$ ) solutions for $\mathbb{C P}^{2}$ and $d P_{1}$. Our interpretation of our solutions for the $d P_{2}$ and $d P_{3}$ case is that they consist of two separated parts. One part represents a D4-brane wrapped around the fuzzy del Pezzo surface, and the other part associated with the node of the unbroken gauge group represents a collection of D0-branes not bound to the D4-brane.

Our construction of fuzzy solutions using oscillators, which is convenient for solving the D-term condition, works if the F-term equations are homogeneous, i.e., they have the same number of chiral fields on both sides of equation as in eq. (3.4) for the $\mathbb{C P}^{2}$ case, and eq. (5.2) for the $d P_{1}$ case. However, since the superpotentials for the $d P_{2}$ and $d P_{3}$ quiver theories contain terms of varying degrees, such as cubic, quartic or quintic superpotential terms etc., many F-term equations become inhomogeneous and hence it is not straightforward to solve the F-term condition simultaneously with the D-term condition by our oscillator method. Therefore, in the above construction of fuzzy $d P_{2}$ and $d P_{3}$ we have deleted many 
arrows to make the F-term equations trivial (namely, the F-term equations are vacuous $0=0$ for Fig. $7 \mathrm{~b}$ and Fig. 8b). We have not yet found a systematic method to delete arrows in the $d P_{2}$ and $d P_{3}$ quiver in such a way that the F-term equations are non-vacuous and at the same time D-term condition is satisfied. It will be nice to construct the most general fuzzy $d P_{2}$ and $d P_{3}$ solutions which will presumably be obtained by deleting arrows according to the Beilinson quiver for $d P_{2}$ and $d P_{3}$ (which is sometimes called Bondal quiver [37,45). We will speculate more about the possible systematic construction of the solutions in the discussion section.

\section{Toward Application to F(uzz) Theory}

So far we have considered classical solutions of the guiver gauge theories on D0branes describing D4-branes wrapped around fuzzy del Pezzo surfaces. In the context of local F-theory GUT model building, it is known that D7-branes should wrap around a del Pezzo surface in order to be able to take the gravity decoupling limit [6]. Therefore, it is tempting to generalize our solutions to D7-branes wrapped on fuzzy del Pezzo surfaces by starting from D3-branes at del Pezzo singularities instead of starting from D0-branes. However, there is a problem of gauge anomaly in the worldvolume theory of D3-branes if we naively generalize our construction of fuzzy del Pezzo surfaces to a solution of the 4-dimensional quiver gauge theory on the fractional D3-branes. In the next subsection we will comment on possible ways to cancel the anomaly. If we put this issue of anomaly aside, our construction of D7-branes wrapped on a fuzzy del Pezzo surface might have an interesting applications in the fuzzy version of the F-theory GUTs 4 ].

\subsection{Note on Anomaly Cancellation}

Since the quiver gauge theory in 4-dimensions is a chiral gauge theory, it has a potential non-Abelian gauge anomaly. For the quiver gauge theory with gauge group $\prod_{i} U\left(n_{i}\right)$ and the adjacency matrix $a_{i j}$ (viz. $a_{i j}$ is the number of chiral multiplets in the representation $\left.\left(n_{i}, \overline{n_{j}}\right)\right)$, the condition for the absence of gauge anomaly at the $i$-th node is 10

$$
\sum_{j}\left(a_{i j}-a_{j i}\right) n_{j}=0
$$

10 This anomaly cancellation condition is closely related to RR-flux conservation 446]. 
If we consider the $\mathbb{C}^{3} / \mathbb{Z}_{3}$ quiver gauge theory with our choice of $n_{i}(3.12)$ as a 4-dimensional gauge theory, this anomaly cancellation condition is not satisfied and hence the gauge symmetry on the fractional D3-brane is anomalous.

At the field theory level, we can cancel this anomaly by adding extra chiral matter fields as "spectator". For instance, in the case of $\mathbb{C}^{3} / \mathbb{Z}_{3}$ quiver gauge theory in Fig. 1a, the theory becomes anomaly free if we choose the number of arrows on each edge to be equal to the rank of gauge group at the node facing that edge. This cancellation of anomaly by adding spectator fields and changing the number of arrows works also for the quiver gauge theories associated with higher del Pezzo surfaces.

In string theory, we cannot arbitrarily add chiral matter fields as above. However, we can add flavor D7-branes which wrap a non-compact cycle in the Calabi-Yau manifold 47,48 to cancel the anomaly. For the case of $\mathbb{C}^{3} / \mathbb{Z}_{3}$ quiver gauge theory, adding flavor D7-branes gives rise to three extra nodes representing flavor symmetry groups, and those flavor nodes are connected to the three nodes of color gauge groups. One can easily check that for the given gauge group $U\left(n_{1}\right) \times U\left(n_{2}\right) \times U\left(n_{3}\right)$ the anomaly is canceled by choosing the rank of the flavor groups appropriately.

The added spectator fields do not play any role in our construction of classical solution since we can simply set those fields to zero in the classical solution. Therefore, we do not have to change the form of our solution even after adding spectator fields to make the quiver gauge theory anomaly free as a 4-dimensional gauge theory.

\section{Discussions}

In this paper, we initiated the study of the classical solutions in quiver gauge theories which represent D-branes wrapped on fuzzy del Pezzo surfaces. There will be many interesting directions for further investigations.

For the fuzzy $\mathbb{C P}^{2}$ solutions, we have seen that our construction is closely related to the Beilinson's construction of stable vector bundles on $\mathbb{C P}^{2}$, and it should be possible to make similar analysis for the fuzzy $d P_{1}$ solutions. On the other hand, in the construction of the fuzzy $d P_{2,3}$ solutions, we had quiver diagrams which differ from the Beilinson quivers. In particular, there was one node which was not included in the diagrams. This is probably because we have picked up a particular simple class of solutions for the F-term condition

in these cases. It is known that brane tilings $49,34,1]$ provides an efficient way to solve the

11 See [50,51] for a review on brane tilings. 
F-term condition, and we may be able to find more general solutions with this technique. In particular, our construction is based on the toric diagrams, and therefore it would be applicable once the F-term condition is rewritten in a form of D-term condition for a gauged linear sigma model describing a toric variety, as has been done using brane tilings. It will also be interesting to investigate whether our solutions with an inclusion of flavor D7-branes to cancel anomaly have a nice description in the language of brane tilings.

In our construction the fuzzy del Pezzo surfaces are described by finite size matrices, whose consequences were emphasized in [4]. If we view these toric del Pezzo surfaces as a fibration of torus over a base space, the base space is made of finite number of points. We have seen that the blow-up at a point in the fuzzy toric del Pezzo surfaces amounts to removing some of the points in the toric diagram. A similar structure has appeared in the quantum foam description of toric Calabi-Yau manifolds [52]. One should note that the physical set up there and in this paper are quite different: The quantum foam describes the fuzziness of the target space-time, whereas the discussions in this paper was about the fuzziness on the worldvolume of D-branes. Keeping this in mind, we may also pursue the similarity in the mathematical structures in both cases. The mathematical results in the study of quantum foam may have interesting physical interpretation in the current setting.

While our construction was based on the toric description, it will be nice to have a generalization of our construction to include non-toric del Pezzo surfaces.

One of our initial motivations for this work was to use D7-branes wrapped on fuzzy del Pezzo surfaces in the phenomenological model buildings, following the idea of [1]. We have presented our preliminary study in this direction in section 6 . If we set aside the problem of gauge anomaly, our construction can be easily generalized to the D3-branes puffed up into D7-branes whose internal 4-dimensional part of the worldvolume wraps around a fuzzy del Pezzo surface. We hope that our construction has useful applications in the fuzzy version of F-theory GUT, or F(uzz) theory. In our approach to F(uzz) theory, we start with a 4-dimensional quiver gauge theory. The fuzzy extra dimensions arise from the VEV of the scalar fields in the four dimensional theory. We found that the KK spectrum is similar to that coming from the usual extra dimensions, but there is a UV cut-off for the KK modes set by the non-commutativity. This opens up a possibility of studying $\mathrm{F}$ (uzz) theory and GUT phenomenology entirely within a four dimensional theory without introducing extra dimensions from the beginning. Our scheme can be summarized as

$$
d=(3+1) \text { quiver } \stackrel{\mathrm{VEV}}{\Longrightarrow} \text { fuzzy } d=(7+1) \stackrel{\mathrm{KK}}{\Longrightarrow} d=(3+1) \text { GUT } .
$$


In this paper, we introduced the curves as data external to the quiver gauge theory, but it may be interesting to realize these curves as non-commutative solitons [53] of the theory. Although the construction in this paper was within the framework of type IIB string theory, we hope that the essential part of our construction also goes through in F-theory. It would be nice to construct more realistic models for the F-theory GUT phenomenology using our construction of fuzzy del Pezzo surfaces. D-branes wrapped on fuzzy del Pezzo surfaces may also have applications in the construction of supersymmetric standard models from D3-branes on del Pezzo surfaces [54,35].

\section{Acknowledgments}

K.F. is supported by the National Center for Theoretical Sciences, Taiwan, R.O.C. He would like to thank Miranda C. N. Cheng, Wu-Yen Chuang and Keijiro Takahashi for the nice lectures on relevant topics at Taiwan String Group. He also thanks Hirotaka Irie, Hiroshi Isono and Pei-Ming Ho for useful discussions. K.O. is supported in part by JSPS Grant-in-Aid for Young Scientists (B) 19740135. He would like to thank Michael Douglas and Jonathan Heckman for discussions during the conference "String Phenomenology 2010" at College de France, Paris. The authors would also like to thank the referee of JHEP for pointing out a subtle point in the calculation of the Yukawa couplings which was overlooked in the earlier version of this paper.

\section{Appendix A. Central Charge of D4-branes Wrapped on $\mathbb{C P}^{2}$}

In this appendix, we review the computation of BPS central charge of D-branes on $\mathbb{C}^{3} / \mathbb{Z}_{3}$ [11]. The relation between the ranks of gauge groups $n_{1,2,3}$ for the quiver diagram in Fig. 1a and the D-brane charge is found by analyzing the monodromy of the period integral at the orbifold point. In terms of the basis $\left(1, t, t_{d}\right)$ of the solution of the PicardFuchs equation with $t_{d} \sim \frac{1}{2} t^{2}+\frac{1}{8}$ near the orbifold point, the monodromy matrix is given by [11:10]

$$
M=\left(\begin{array}{ccc}
1 & 0 & 0 \\
-\frac{1}{2} & -2 & -3 \\
\frac{1}{2} & 1 & 1
\end{array}\right)
$$

which satisfies $M^{3}=1$. Applying this matrix to $\left(1, t, t_{d}\right)$, we find

$$
M\left(\begin{array}{c}
1 \\
t \\
t_{d}
\end{array}\right)=\left(\begin{array}{c}
1 \\
-\frac{1}{2}-2 t-3 t_{d} \\
\frac{1}{2}+t+t_{d}
\end{array}\right), \quad M^{2}\left(\begin{array}{c}
1 \\
t \\
t_{d}
\end{array}\right)=\left(\begin{array}{c}
1 \\
-1+t+3 t_{d} \\
\frac{1}{2}-t-2 t_{d}
\end{array}\right) .
$$


By identifying the periods in the fractional brane basis

$$
\Pi_{1}=\frac{1}{2}+t+t_{d}, \quad \Pi_{2}=\frac{1}{2}-t-2 t_{d}, \quad \Pi_{3}=t_{d},
$$

the central charge becomes

$$
Z=\sum_{i=1}^{3} n_{i} \Pi_{i}=\frac{n_{1}+n_{2}}{2}-\left(n_{2}-n_{1}\right) t+\left(n_{1}-2 n_{2}+n_{3}\right) t_{d} .
$$

This gives the relation between $n_{i}$ and the D-brane charge (3.21).

This relation also follows from the Beilinson quiver for $\mathbb{C P}^{2}$. The Beilinson quiver is associated with the sequence

$$
\mathbb{C}^{n_{1}} \otimes \Omega^{2}(2) \stackrel{X}{\longrightarrow} \mathbb{C}^{n_{2}} \otimes \Omega^{1}(1) \stackrel{Y}{\longrightarrow} \mathbb{C}^{n_{3}} \otimes \mathcal{O}
$$

and the central charge $Z$ corresponding to this configuration is

$$
\begin{aligned}
Z & =n_{1} \operatorname{ch} \Omega^{2}(2)-n_{2} \operatorname{ch} \Omega^{1}(1)+n_{3} \operatorname{ch} \mathcal{O} \\
& =n_{1} e^{-\omega}-n_{2}\left(3-e^{\omega}\right)+n_{3} \\
& =n_{1}-2 n_{2}+n_{3}+\left(n_{2}-n_{1}\right) \omega+\frac{n_{1}+n_{2}}{2} \omega^{2} .
\end{aligned}
$$

Here we have used

$$
\Omega^{2}=K_{\mathbb{P}^{2}}=\mathcal{O}(-3), \quad \Omega^{2}(2)=\mathcal{O}(-3) \otimes \mathcal{O}(2)=\mathcal{O}(-1)
$$

and the relation $\operatorname{ch} \Omega^{1}(1)=3-e^{\omega}$ used in (A.6) comes from the exactness of the (dual) Euler sequence

$$
\begin{gathered}
0 \longrightarrow \Omega_{\mathbb{P}(V)}(1) \longrightarrow V^{*} \otimes \mathcal{O} \longrightarrow \mathcal{O}(1) \longrightarrow 0 \\
\operatorname{ch} \Omega^{1}(1)=\operatorname{ch}\left(V^{*} \otimes \mathcal{O}\right)-\operatorname{ch} \mathcal{O}(1)=3-e^{\omega} .
\end{gathered}
$$

For the $k$ copies of D4-brane solutions (3.27), the D-brane charges are given just by $k$ times the above. More generally, one can embed D4-brane solutions with different ranks in a block diagonal form. In this case, the D-brane charges are given just by the sum of the D-brane charges in each block. Sometimes the same gauge group allows several different combinations for the rank of the D4-brane solutions, but the total D-brane charges remain the same. 
Similarly, we can associate a sequence for the D2-brane wrapped on $\mathbb{C P}^{1}$ in the $A_{1}$ quiver theory

$$
\mathbb{C}^{n_{1}} \otimes \Omega^{1}(1) \stackrel{X}{\longrightarrow} \mathbb{C}^{n_{2}} \otimes \mathcal{O}
$$

The central charge for this configuration is

$$
\begin{aligned}
Z & =-n_{1} \operatorname{ch} \Omega^{1}(1)+n_{2} \operatorname{ch} \mathcal{O} \\
& =-n_{1} \operatorname{ch} \mathcal{O}(-1)+n_{2} \operatorname{ch} \mathcal{O} \\
& =-n_{1} e^{-\omega}+n_{2} .
\end{aligned}
$$

For our choice of Chan-Paton spaces (2.6), we find

$$
Z=-(N+1) e^{-\omega}+N+2=1+(N+1) \omega
$$

This implies that a magnetic flux $\frac{F}{2 \pi}=(N+1) \omega$ is threading the $\mathbb{C P}^{1}$ part of the D2brane worldvolume, and the quantized sections of the line bundle $\mathcal{O}_{\mathbb{P}^{1}}(N+1)$ is identified with the Fock space $\mathbb{C}^{n_{2}}=\mathcal{F}_{N+1}^{(2)}$. More physically, those states correspond to the lowest Landau levels on $S^{2}$ in the presence of magnetic field.

\section{Appendix B. Intersections of Curves on Fuzzy $\mathbb{C P}^{2}$}

In this appendix, we will consider intersections of curves on fuzzy $\mathbb{C P}^{2}$. The curves represent the intersection of other D7-branes with our fuzzy D7-branes. In this paper, we treat these curves as data external to the theory on the fuzzy D7-branes. We will follow the analogous computation for the fuzzy $\mathbb{P}^{1} \times \mathbb{P}^{1}$ considered in [4].

In terms of the inhomogeneous coordinates $u=z_{1} / z_{3}, v=z_{2} / z_{3}$ of $\mathbb{C P}^{2}$ on the patch $z_{3} \neq 0$, the configuration of matter curves can be modeled by the Higgsing of $U(3)$ gauge theory on the D7-branes intersecting with the fuzzy D7-branes down to $U(1)^{3}$ according to the background VEV 四

$$
\Phi_{0}=\left(\begin{array}{ccc}
u & 0 & 0 \\
0 & 0 & 0 \\
0 & 0 & \beta v
\end{array}\right) .
$$

Here, we have introduced a complex number $\beta$ which parametrizes the direction of the matter curve. The three matter curves of this configuration correspond to the enhancement 
of unbroken symmetry $U(1)^{3} \rightarrow U(1) \times U(2)$ at $u=0, v=0$ and $u=\beta v$. In terms of the homogeneous coordinates $\left[z_{1}: z_{2}: z_{3}\right]$, the three matter curves are given by

$$
\begin{aligned}
\mathbb{C P}_{\mathrm{I}}^{1} & =\left\{z_{1}=0\right\} \\
\mathbb{C P}_{\mathrm{II}}^{1} & =\left\{z_{2}=0\right\} \\
\mathbb{C P}_{b}^{1} & =\left\{z_{1}=\beta z_{2}\right\}
\end{aligned}
$$

Yukawa couplings come from the intersection of the matter curves. In the commutative case, the intersection of two curves is a single point for all pair of the curves in (B.2)

$$
\mathbb{C P}_{\mathrm{I}}^{1} \cap \mathbb{C P}_{\mathrm{II}}^{1}=\mathbb{C P}_{\mathrm{I}}^{1} \cap \mathbb{C P}_{b}^{1}=\mathbb{C P}_{\mathrm{II}}^{1} \cap \mathbb{C P}_{b}^{1}=[0: 0: 1]
$$

However, the intersection of those curves is different from (B.3) in the fuzzy setup as we will see below.

On fuzzy $\mathbb{C P}^{2}$, these matter curves correspond to subspaces of the Fock space $\mathcal{F}_{N}^{(3)}$ annihilated by $a_{1}, a_{2}$ and $a_{1}-\beta a_{2}$, respectively

$$
\begin{aligned}
& \mathcal{F}\left(\mathbb{C P}_{\mathrm{I}}^{1}\right)=\operatorname{ker}\left(a_{1}\right)=\left\{\left|0, m_{2}, m_{3}\right\rangle, \quad m_{2}+m_{3}=N\right\} \\
& \mathcal{F}\left(\mathbb{C P}_{\mathrm{II}}^{1}\right)=\operatorname{ker}\left(a_{2}\right)=\left\{\left|m_{1}, 0, m_{3}\right\rangle, \quad m_{1}+m_{3}=N\right\} \\
& \mathcal{F}\left(\mathbb{C P}_{b}^{1}\right)=\operatorname{ker}\left(b_{1}\right)=\left\{\left(b_{2}^{\dagger} a_{3}\right)^{m}|0,0, N\rangle, \quad 0 \leq m \leq N\right\},
\end{aligned}
$$

where we have defined $b_{1}, b_{2}$ as the linear combination of $a_{1}$ and $a_{2}$

$$
b_{1}=\frac{a_{1}-\beta a_{2}}{\sqrt{1+|\beta|^{2}}}, \quad b_{2}=\frac{\bar{\beta} a_{1}+a_{2}}{\sqrt{1+|\beta|^{2}}}, \quad\left[b_{i}, b_{j}^{\dagger}\right]=\delta_{i j}
$$

In the fuzzy setup, the Yukawa coupling is computed by sandwiching certain operators between projection operators corresponding to the above spaces $\mathcal{F}\left(\mathbb{C P}_{\alpha}^{1}\right)(\alpha=\mathrm{I}, \mathrm{II}, b)$

$$
\begin{aligned}
\mathcal{P}_{\mathrm{I}} & =\sum_{m=0}^{N}|0, m, N-m\rangle\langle 0, m, N-m| \\
\mathcal{P}_{\mathrm{II}} & =\sum_{m=0}^{N}|m, 0, N-m\rangle\langle m, 0, N-m| \\
\mathcal{P}_{b} & =\sum_{m=0}^{N}|0, m, N-m\rangle_{b}{ }_{b}\langle 0, m, N-m| .
\end{aligned}
$$


Here $|0, m, N-m\rangle_{b}$ denote the orthogonal states in $\mathcal{F}\left(\mathbb{C P}_{b}^{1}\right)$, i.e., they are the Fock states of the $b_{1}, b_{2}, a_{3}$ oscillators

$$
\left|m_{1}, m_{2}, m_{3}\right\rangle_{b}=\frac{\left(b_{1}^{\dagger}\right)^{m_{1}}\left(b_{2}^{\dagger}\right)^{m_{2}}\left(a_{3}^{\dagger}\right)^{m_{3}}}{\sqrt{m_{1} ! m_{2} ! m_{3} !}}|0,0,0\rangle
$$

The state $|0, m, N-m\rangle_{b}$ is written in the original Fock states of $a_{1}, a_{2}, a_{3}$ oscillators as

$$
|0, m, N-m\rangle_{b}=\sum_{k=0}^{m} \frac{\beta^{k}}{\sqrt{\left(1+|\beta|^{2}\right)^{m}}} \sqrt{\frac{m !}{k !(m-k !)}}|k, m-k, N-m\rangle .
$$

From this expression we can read off the overlap between $|0, m, N-m\rangle_{b}$ and the basis of $\mathcal{F}\left(\mathbb{C P}_{\mathrm{I}}^{1}\right), \mathcal{F}\left(\mathbb{C P}_{\text {II }}^{1}\right)$ as

$$
\begin{aligned}
\left\langle 0, m, N-m \mid 0, m^{\prime}, N^{\prime}-m^{\prime}\right\rangle_{b} & =\left(\frac{1}{\sqrt{1+|\beta|^{2}}}\right)^{m} \delta_{m, m^{\prime}} \delta_{N, N^{\prime}} \\
\left\langle m, 0, N-m \mid 0, m^{\prime}, N^{\prime}-m^{\prime}\right\rangle_{b} & =\left(\frac{\beta}{\sqrt{1+|\beta|^{2}}}\right)^{m} \delta_{m, m^{\prime}} \delta_{N, N^{\prime}}
\end{aligned}
$$

This means that the states on $\mathbb{C P}_{b}^{1}$ can interpolate the states on $\mathbb{C P}_{\mathrm{I}}^{1}$ and $\mathbb{C P}_{\mathrm{II}}^{1}$. In particular, the product of projection operators $\mathcal{P}_{b}$ and $\mathcal{P}_{\mathrm{I}, \mathrm{II}}$ is different from the projection to the classical intersection point $[0: 0: N](\overline{\mathrm{B} .3})$ :

$$
\begin{aligned}
\mathcal{P}_{\mathrm{I}} \mathcal{P}_{\mathrm{II}} & =|0,0, N\rangle\langle 0,0, N| \\
\mathcal{P}_{\mathrm{I}} \mathcal{P}_{b} & =\sum_{m=0}^{N}\left(\frac{1}{\sqrt{1+|\beta|^{2}}}\right)^{m}|0, m, N-m\rangle_{b}\langle 0, m, N-m| \\
\mathcal{P}_{\mathrm{II}} \mathcal{P}_{b} & =\sum_{m=0}^{N}\left(\frac{\beta}{\sqrt{1+|\beta|^{2}}}\right)^{m}|m, 0, N-m\rangle_{b}\langle 0, m, N-m| .
\end{aligned}
$$

It is interesting to observe that the intersection of the fuzzy curves $\mathbb{C P}_{b}^{1}$ and $\mathbb{C P}_{\mathrm{I}, \mathrm{II}}^{1}$ samples all the "fuzzy points" on $\mathbb{C P}_{b}^{1}$, i.e. all the states in $\mathcal{F}\left(\mathbb{C P}_{b}^{1}\right)[\mathbb{4}$. This is different from the situation in the $\mathbb{P}^{1} \times \mathbb{P}^{1}$ example considered in 4 .

If we naively generalize the computation of Yukawa couplings on $\mathbb{P}^{1} \times \mathbb{P}^{1}$ studied in [4] to our fuzzy $\mathbb{C P}^{2}$ case, we seem to get non-trivial Yukawa texture with a hierarchical factor like $\left(\beta / \sqrt{1+|\beta|^{2}}\right)^{m}$ coming from the overlap of $\mathbb{C P}_{\mathrm{I}, \mathrm{II}}^{1}$ and $\mathbb{C P}_{b}^{1}$ in $(\mathbb{B} .9)$. However, in the above the curves representing the intersections with other D7-branes were introduced as external inputs to the quiver gauge theory, and it remains to be checked whether those 
curves correctly describe the intersections with other D7-branes. Accordingly, there are some subtle points in applying the naive generalization of the prescription given in 4 to our case.12 It might be the case that our non-holomorphic non-commutativity does not alter the result of the holomorphic calculation of the Yukawa couplings in [2], in particular, the rank one theorem in the commutative case. We would like to clarify this point in the near future and see if the above interpolating property of $\mathbb{C P}_{b}^{1}$ has interesting physical consequences in the texture of the Yukawa couplings.

12 We would like to thank the referee of JHEP for pointing out some issues. 


\section{References}

[1] N. Seiberg and E. Witten, "String theory and noncommutative geometry," JHEP 9909, 032 (1999) arXiv:hep-th/9908142.

[2] S. Cecotti, M. C. N. Cheng, J. J. Heckman and C. Vafa, "Yukawa Couplings in Ftheory and Non-Commutative Geometry," arXiv:0910.0477 [hep-th].

[3] F. Marchesano and L. Martucci, "Non-perturbative effects on seven-brane Yukawa couplings," Phys. Rev. Lett. 104, 231601 (2010) [arXiv:0910.5496 [hep-th]].

[4] J. J. Heckman and H. Verlinde, "Evidence for F(uzz) Theory," arXiv:1005.3033 [hepth].

[5] R. Donagi and M. Wijnholt, "Model Building with F-Theory," arXiv:0802.2969 [hepth].

[6] C. Beasley, J. J. Heckman and C. Vafa, "GUTs and Exceptional Branes in F-theory I," JHEP 0901, 058 (2009) [arXiv:0802.3391 [hep-th]]; "GUTs and Exceptional Branes in F-theory - II: Experimental Predictions," JHEP 0901, 059 (2009) arXiv:0806.0102 [hep-th]].

[7] J. J. Heckman, "Particle Physics Implications of F-theory," arXiv:1001.0577 [hep-th].

[8] D. Berenstein and R. G. Leigh, "Non-commutative Calabi-Yau manifolds," Phys. Lett. B 499, 207 (2001) arXiv:hep-th/0009209.

[9] D. E. Diaconescu, M. R. Douglas and J. Gomis, "Fractional branes and wrapped branes," JHEP 9802, 013 (1998) arXiv:hep-th/9712230.

[10] D. E. Diaconescu and J. Gomis, "Fractional branes and boundary states in orbifold theories," JHEP 0010, 001 (2000) arXiv:hep-th/9906242.

[11] M. R. Douglas, B. Fiol and C. Romelsberger, "The spectrum of BPS branes on a noncompact Calabi-Yau," JHEP 0509, 057 (2005) arXiv:hep-th/0003263.

[12] S. Franco, A. Hanany, F. Saad and A. M. Uranga, "Fractional Branes and Dynamical Supersymmetry Breaking," JHEP 0601, 011 (2006) arXiv:hep-th/0505040].

[13] A. Butti, "Deformations of toric singularities and fractional branes," JHEP 0610, 080 (2006) arXiv:hep-th/0603253.

[14] M. R. Douglas and G. W. Moore, "D-branes, Quivers, and ALE Instantons," arXiv:hep-th/9603167.

[15] M. R. Douglas, B. R. Greene and D. R. Morrison, "Orbifold resolution by D-branes," Nucl. Phys. B 506, 84 (1997) arXiv:hep-th/9704151.

[16] C. Beasley, B. R. Greene, C. I. Lazaroiu and M. R. Plesser, "D3-branes on partial resolutions of abelian quotient singularities of Calabi-Yau threefolds," Nucl. Phys. B 566, 599 (2000) arXiv:hep-th/9907186.

[17] Y. Kitazawa, "Matrix models in homogeneous spaces," Nucl. Phys. B 642, 210 (2002) arXiv:hep-th/0207115. 
[18] B. Janssen, Y. Lozano and D. Rodriguez-Gomez, "Giant gravitons and fuzzy CP(2)," Nucl. Phys. B 712, 371 (2005) arXiv:hep-th/0411181.

[19] D. Berenstein and R. Corrado, "Matrix theory on ALE spaces and wrapped membranes," Nucl. Phys. B 529, 225 (1998) arXiv:hep-th/9803048.

[20] H. Nastase, C. Papageorgakis and S. Ramgoolam, "The fuzzy $S^{2}$ structure of M2M5 systems in ABJM membrane theories," JHEP 0905, 123 (2009) arXiv:0903.3966 [hep-th]].

[21] J. Maldacena and D. Martelli, "The unwarped, resolved, deformed conifold: fivebranes and the baryonic branch of the Klebanov-Strassler theory," JHEP 1001, 104 (2010) |arXiv:0906.0591 [hep-th]].

[22] A. A. Beilinson, "Coherent sheaves on $\mathbb{P}^{n}$ and problems of linear algebra," Funct. Anal. Appl. 12 (1978), 214-216.

[23] A. Dymarsky, I. R. Klebanov and N. Seiberg, "On the moduli space of the cascading $S U(M+p) \times S U(p)$ gauge theory," JHEP 0601, 155 (2006) arXiv:hep-th/0511254.

[24] I. R. Klebanov and E. Witten, "Superconformal field theory on threebranes at a Calabi-Yau singularity," Nucl. Phys. B 536, 199 (1998) arXiv:hep-th/9807080].

[25] A. Iqbal, A. Neitzke and C. Vafa, "A mysterious duality," Adv. Theor. Math. Phys. 5, 769 (2002) arXiv:hep-th/0111068.

[26] C. Saemann, "Fuzzy Toric Geometries," JHEP 0802, 111 (2008) arXiv:hep-th/0612173

[27] G. Alexanian, A. P. Balachandran, G. Immirzi and B. Ydri, "Fuzzy CP(2)," J. Geom. Phys. 42, 28 (2002) arXiv:hep-th/0103023.

[28] U. Carow-Watamura, H. Steinacker and S. Watamura, "Monopole bundles over fuzzy complex projective spaces," J. Geom. Phys. 54, 373 (2005) arXiv:hep-th/0404130.

[29] A. Ikeda and Y. Taniguchi, "Spectra and Eigenforms of the Laplacian on $S^{n}$ and $P^{n}(C)$," Osaka J. Math. 15 (1978) 515.

[30] V. Braun, T. Brelidze, M. R. Douglas and B. A. Ovrut, "Eigenvalues and Eigenfunctions of the Scalar Laplace Operator on Calabi-Yau Manifolds," JHEP 0807, 120 (2008) [arXiv:0805.3689 [hep-th]].

[31] H. Grosse and A. Strohmaier, "Noncommutative geometry and the regularization problem of 4D quantum field theory," Lett. Math. Phys. 48, 163 (1999) arXiv:hepth/9902138].

[32] B. Feng, A. Hanany and Y. H. He, "D-brane gauge theories from toric singularities and toric duality," Nucl. Phys. B 595, 165 (2001) arXiv:hep-th/0003085.

[33] C. E. Beasley and M. R. Plesser, "Toric duality is Seiberg duality," JHEP 0112, 001 (2001) arXiv:hep-th/0109053.

[34] S. Franco, A. Hanany, K. D. Kennaway, D. Vegh and B. Wecht, "Brane Dimers and Quiver Gauge Theories," JHEP 0601, 096 (2006) arXiv:hep-th/0504110]. 
[35] S. Krippendorf, M. J. Dolan, A. Maharana and F. Quevedo, "D-branes at Toric Singularities: Model Building, Yukawa Couplings and Flavour Physics," arXiv:1002.1790 [hep-th].

[36] P. S. Aspinwall and I. V. Melnikov, "D-branes on vanishing del Pezzo surfaces," JHEP 0412, 042 (2004) arXiv:hep-th/0405134.

[37] A. I. Bondal, "Helices, representations of quivers and Koszul algebras," in A. N. Rudakov" et al. Helices and vector bundles 75-95, London Mathematical Society Lecture Note Series 148, Cambridge University Press, 1990.

[38] S. Govindarajan and T. Jayaraman, "D-branes, exceptional sheaves and quivers on Calabi-Yau manifolds: From Mukai to McKay," Nucl. Phys. B 600, 457 (2001) arXiv:hep-th/0010196.

[39] A. Tomasiello, "D-branes on Calabi-Yau manifolds and helices," JHEP 0102, 008 (2001) arXiv:hep-th/0010217.

[40] C. P. Herzog, "Seiberg duality is an exceptional mutation," JHEP 0408, 064 (2004) arXiv:hep-th/0405118.

[41] A. Hanany, C. P. Herzog and D. Vegh, "Brane tilings and exceptional collections," JHEP 0607, 001 (2006) arXiv:hep-th/0602041.

[42] B. Feng, A. Hanany and Y. H. He, "Phase structure of D-brane gauge theories and toric duality," JHEP 0108, 040 (2001) arXiv:hep-th/0104259].

[43] D. Berenstein and M. R. Douglas, "Seiberg duality for quiver gauge theories," arXiv:hep-th/0207027.

[44] B. Feng, A. Hanany, Y. H. He and A. M. Uranga, "Toric duality as Seiberg duality and brane diamonds," JHEP 0112, 035 (2001) arXiv:hep-th/0109063.

[45] N. J. Proudfoot and A. Bergman, "Moduli spaces for Bondal quivers," Pacific J. Math. 237, 201 (2008) arXiv:math/0512166.

[46] F. Cachazo, B. Fiol, K. A. Intriligator, S. Katz and C. Vafa, "A geometric unification of dualities," Nucl. Phys. B 628, 3 (2002) arXiv:hep-th/0110028.

[47] S. Franco and A. M. Uranga, "Dynamical SUSY breaking at meta-stable minima from D-branes at obstructed geometries," JHEP 0606, 031 (2006) arXiv:hep-th/0604136.

[48] Y. Imamura, K. Kimura and M. Yamazaki, "Anomalies and O-plane charges in orientifolded brane tilings," JHEP 0803, 058 (2008) [arXiv:0801.3528 [hep-th]].

[49] A. Hanany and K. D. Kennaway, "Dimer models and toric diagrams," arXiv:hepth/0503149.

[50] K. D. Kennaway, "Brane Tilings," Int. J. Mod. Phys. A 22, 2977 (2007) arXiv:0706.1660 [hep-th]].

[51] M. Yamazaki, "Brane Tilings and Their Applications," Fortsch. Phys. 56, 555 (2008) arXiv:0803.4474 [hep-th]].

[52] A. Iqbal, N. Nekrasov, A. Okounkov and C. Vafa, "Quantum foam and topological strings," JHEP 0804, 011 (2008) arXiv:hep-th/0312022. 
[53] R. Gopakumar, S. Minwalla and A. Strominger, "Noncommutative solitons," JHEP 0005, 020 (2000) arXiv:hep-th/0003160.

[54] H. Verlinde and M. Wijnholt, "Building the Standard Model on a D3-brane," JHEP 0701, 106 (2007) arXiv:hep-th/0508089. 